\title{
Metamodeling and On-Line Clustering for Loss-of-Flow Accident Precursors Identification in a Superconducting Magnet Cryogenic Cooling Circuit
}

\author{
Vincenzo Destino $^{1}$, Nicola Pedroni ${ }^{1, *(\mathbb{D})}$, Roberto Bonifetto ${ }^{1}$ (D) Francesco Di Maio ${ }^{2}$ (D) Laura Savoldi $^{1}(\mathbb{D})$ \\ and Enrico Zio 2,3,4 \\ 1 Dipartimento Energia, Politecnico di Torino, Corso Duca degli Abruzzi 24, 10129 Torino, Italy; \\ vincenzo.destino@studenti.polito.it (V.D.); roberto.bonifetto@polito.it (R.B.); laura.savoldi@polito.it (L.S.) \\ 2 Energy Department, Politecnico di Milano, Via La Masa 34, 20156 Milano, Italy; \\ francesco.dimaio@polimi.it (F.D.M.); enrico.zio@polimi.it (E.Z.) \\ 3 Centre de Recherche sur les Risques et les Crises (CRC), MINES ParisTech/PSL Université Paris, \\ 75272 Paris, France \\ 4 Department of Nuclear Engineering, Kyung Hee University, Seoul 17104, Korea \\ * Correspondence: nicola.pedroni@polito.it
}

check for updates

Citation: Destino, V.; Pedroni, N.; Bonifetto, R.; Di Maio, F.; Savoldi, L.; Zio, E. Metamodeling and On-Line Clustering for Loss-of-Flow Accident Precursors Identification in a Superconducting Magnet Cryogenic Cooling Circuit. Energies 2021, 14, 5552. https://doi.org/10.3390/ en14175552

Academic Editor: Hiroshi Sekimoto

Received: 14 July 2021

Accepted: 31 August 2021

Published: 5 September 2021

Publisher's Note: MDPI stays neutral with regard to jurisdictional claims in published maps and institutional affiliations.

Copyright: (c) 2021 by the authors. Licensee MDPI, Basel, Switzerland. This article is an open access article distributed under the terms and conditions of the Creative Commons Attribution (CC BY) license (https:/ / creativecommons.org/licenses/by/ $4.0 /)$.

\begin{abstract}
In the International Thermonuclear Experimental Reactor, plasma is magnetically confined with Superconductive Magnets (SMs) that must be maintained at the cryogenic temperature of $4.5 \mathrm{~K}$ by one or more Superconducting Magnet Cryogenic Cooling Circuits (SMCCC). To guarantee cooling, Loss-of-Flow Accidents (LOFAs) in the SMCCC are to be avoided. In this work, we develop a three-step methodology for the prompt detection of LOFA precursors (i.e., those combinations of component failures causing a LOFA). First, we randomly generate accident scenarios by Monte Carlo sampling of the failures of typical SMCCC components and simulate the corresponding transient system response by a deterministic thermal-hydraulic code. In this phase, we also employ quickrunning Proper Orthogonal Decomposition (POD)-based Kriging metamodels, adaptively trained to reproduce the output of the long-running code, to decrease the computational time. Second, we group the generated scenarios by a Spectral Clustering (SC) employing the Fuzzy C-Means (FCM), in order to identify the main patterns of system evolution towards abnormal states (e.g., a LOFA). Third, we develop an On-line Supervised Spectral Clustering (OSSC) technique to associate time-varying parameters measured during plant functioning to one of the prototypical groups obtained, which may highlight the related LOFA precursors (in terms of SMCCC components failures). We apply the proposed technique to the simplified model of a cryogenic cooling circuit of a single module of the ITER Central Solenoid Magnet (CSM). The framework developed promptly detects 95\% of LOFA events and around $80 \%$ of the related precursors.
\end{abstract}

Keywords: ITER Central Solenoid Magnet; cryogenic cooling circuit; Loss-of-Flow Accident; precursors; Spectral Clustering; adaptive Kriging meta-model; Proper Orthogonal Decomposition

\section{Introduction}

The International Thermonuclear Experimental Reactor (ITER) will employ a plasma of Deuterium and Tritium to produce a net energy output by means of thermonuclear fusion reactions for the first time [1]. Different low-critical temperature (LTS) Superconducting Magnets (SMs) are used to confine and shape the plasma, and to drive the plasma current [2]: eighteen Toroidal Field (TF) coils, six Poloidal Field (PF) coils and one Central Solenoid (CS) coil, respectively (the eighteen Correction Coils (CCs) in ITER do not have instead superconductive properties). Each of the six Central Solenoid Modules (CSMs), which compose the CS, must sustain fast currents ramps up to high values of $\sim 40 \mathrm{kA}$ in order to generate the rated plasma current: in such extreme conditions, ohmic heating must be nullified by preserving the magnets superconductive properties [3]. While AC 
losses in the SC cables (due to the magnetic field variation) and parasitic heat load tend to rise the temperature, appropriate cooling is ensured by a Superconducting Magnet Cryogenic Cooling Circuit (SMCCC), where Supercritical Helium (SHe) flows at $4.5 \mathrm{~K}$ and 0.5-0.6 MPa. The heat removed from the magnet is transferred within the SMCCC to a thermal buffer constituted by a bath with liquid Helium (LHe) in equilibrium with its vapor $[4,5]$. Similar Cryogenic Circuits are installed for the TF and PF coils.

The safety of ITER (and of other nuclear fusion systems, like the European Demonstration Power Plant (EU DEMO)) needs to be verified and proved by a rigorous assessment of the system's response to operational transients and accidental conditions, and the related (safety) gaps and issues need to be underlined [6-11]. Actually, we must avoid the plant operators, the people, and the environment being contaminated by radioactive agents, e.g., tritium and other materials activated by the neutrons generated by the nuclear fusion reactions [12-15]. Additionally, the protection and integrity of the superconducting magnet system must be guaranteed, due to its huge cost [16], as well as due to the need to ensure at least a lifetime equal to that of the nuclear fusion plant itself. The relevance of these considerations is testified by the flourishing recent literature concerning the detailed modeling of the behavior of fusion systems under a large variety of abnormal and accidental conditions. For example, in ref. [17] an in-vessel Loss-Of-Coolant Accident (LOCA) is analyzed for the EU DEMO. Instead, in refs. [18-22] in-box and ex-vessel LOCAs, respectively, are studied for the ITER facility; also, in ref. [23] the effects of a pipe break (and the consequent loss of coolant) in the ITER secondary cooling water system are assessed. Loss-of-Flow Accidents (LOFAs) in ITER current leads prototypes are modeled and tested in refs. [24,25], while studies on Loss-Of-Vacuum Accidents (LOVAs) in the ITER vacuum vessel can be found in ref. [26]. Finally, an analysis of the plasma quench propagation tests in the ITER TF insert coil is developed in ref. [27].

Within this broad framework, here we are particularly concerned with Loss-of-Flow Accidents (LOFAs). Actually, a LOFA in a SMCCC represents a major issue because it may jeopardize the SC magnet cooling capability. In case of a LOFA, the CS temperature and pressure may escalate rapidly due to the $\mathrm{AC}$ losses and a loss of the superconductivity (a "quench") could be initiated. If the quench propagates, when the temperature and the pressure exceed $150 \mathrm{~K}$ and $25 \mathrm{MPa}$, respectively, the structural integrity and superconductive properties of the CS could be lost [28-32].

In this paper, we elaborate an automatic, three-step data-driven technique to promptly identify patterns of (time-varying) parameters measured during plant functioning and to reveal LOFA precursors (i.e., combinations of SMCCC components failures) [33,34]. First, we create a "database" of accident scenarios by repeated Monte Carlo Sampling (MCS) of the SMCCC components failures and the simulation of the corresponding transient system response by the deterministic thermal-hydraulic Cryogenic Circuit Conductor and Coil (4C) code [35]. Since this procedure requires a huge computational cost, Kriging metamodels [36] are employed as fast-running "surrogates" to reproduce the behavior of the mechanistic code with a reduced computational time. In particular, we first adopt the Adaptive Kriging-Monte Carlo Sampling (AK-MCS) method to progressively refine the accuracy of the metamodels in reproducing the "critical" system configurations of our interest (i.e., the LOFA conditions) [37-41]. Then, we construct Proper Orthogonal Decomposition (POD)-based Kriging metamodels [42,43] to quickly simulate a large number of (new) time-varying signals without resorting to the original long-running $4 \mathrm{C}$ code: this allows enriching the database of accidental scenarios at a negligible computational cost. In the second step, we employ a Spectral Clustering (SC) algorithm based on the Fuzzy C-Means (FCM) [44] to group similar scenarios together, which allows typifying the main patterns of the system evolution towards failure configurations (e.g., a LOFA). By so doing, we can reveal the "prototypes" of component failure modes (i.e., the precursors) that most likely drive the system to abnormal conditions $[45,46]$. Thirdly, we exploit the information collected within an On-line Supervised Spectral Clustering (OSSC) to timely associate new 
developing scenarios (measured during plant functioning) to the proper clusters and to identify the respective LOFA precursors [47].

The LOFA in ITER magnets has already been studied with a deterministic approach in ref. [31], while a first probabilistic assessment has been proposed in ref. [28] for a sub-size SMCCC tailored for the cooling of a single CSM. In ref. [48], some of the authors of this paper have proposed a framework combining visual maps and a preliminary version of the OSSC algorithm [47] to carry out a more extensive analysis of the same test case of ref. [28], involving LOFA precursor identification in the cooling circuit of the ITER CS magnets. One of the main limitations of [48] was the adoption of a relatively small number of simulated scenarios (i.e., 83) to train the OSSC algorithm and to build the visual maps in order to limit the overall computational cost of the analysis. This was shown to impair the effectiveness of the method, leading, e.g., to erroneous detection of LOFA precursors in some scenarios with no LOFA and to the identification as precursors of some components that had not actually failed. These errors are on the conservative side but may reduce the availability of the machine, due, e.g., to unnecessary inspections following the precursor identification. To reduce such over-identification, the following applicative and methodological contributions are introduced in the present paper with respect to ref. [48]:

- POD-based Kriging metamodels are employed for the first time to simulate-at a reduced computational cost-a large number of time-varying (possibly accidental) behavior of a machine for nuclear fusion;

- Classical techniques available for the post-processing of the clusters produced by the FCM-based SC method [44-46] are originally tailored to identify both the LOFA occurrence times and the components failure modes;

- The version of the OSSC algorithm here used to timely identify LOFA precursors during the development of a new accident scenario is proposed for the first time in this work.

It is worth mentioning that a huge variety of (data-driven, analytical-model-based, and deep-knowledge-based) approaches has been proposed in the open literature for the timely detection and diagnosis of faults in several fields of modern engineering. See refs. [49-52] for interesting and complete reviews. For example, in the field of online monitoring of rotating machinery, a self-adaptable approach based on dynamically evolving feature selection is introduced in ref. [53]. Additionally, in ref. [54] the authors develop a compacted object sample extraction method based on unlabeled data for fault diagnosis in evolving environments. Finally, in ref. [55] detection and diagnosis of anomalies are performed by a combination of Convolutional Long Short-Term Memory, Fast Fourier and continuous wavelet transforms. In the area of energy engineering, a recent example can be found in ref. [56], where evolutionary classification trees and adaptive symbolic aggregate approximation processes are combined to identify anomalous patterns of energy consumption in buildings. Additionally, in ref. [57] a never-ending learning method (based on dendrograms and 1-nearest-neighbor classifiers) is developed for online diagnosis of different types of faults in a gas turbine oil system operating in dynamically evolving environments. In the nuclear field (which is of particular interest to the present paper), several techniques have been employed for the early identification and diagnosis of accidents in fission systems, including: classical neural network architectures and Bayesian statistics for identifying LOCA events in a pressurized heavy water reactor [58]; deep neural networks for the fault detection and remaining useful life prediction of solenoid operated valves [59] and for online monitoring of the (modular) Integrated Pressurized Water Reactor IP-200 [60]; (Kernel) Principal Component Analysis combined with clustering for anomaly detection and isolation in an advanced heavy water reactor [61] and for spotting pipe ruptures in the cooling system of a pressurized light-water reactor [62]; particle filters embedded with neural networks to detect very small-break LOCAs in pressurized water reactors [63]; Auto-Associative Kernel Regression for early warnings about the water level of a pressurizer, on the moisture separator and reheater temperature transmitters and on environmental influences in real nuclear power plants of the Korea Hydro \& Nuclear 
Power Co., Ltd. (KHNP) (Central Research Institute, KHNP, 70, 1312-gil, Yuseong-daero, Yuseong-gu, Daejeon 34101, Republic of Korea) [64]; Bayesian Networks for the modelbased diagnosis in a single-phase heat exchanger [65]; Support Vector Machines combined with Gaussian Process Regression for the transient analysis of seven different (normal and accidental) conditions (LOCAs, load rejection, steam generator rupture, etc.) in a simulated nuclear plant [66]; incremental learning and reconciliation of different clustering approaches by unsupervised schemes applied to a fleet of nuclear power plant turbines during shut-down transients [67]. While acknowledging this wide and diversified framework of algorithms and applications, it is important to notice that to the best of the authors' knowledge: (i) the structured, integrated combination of advanced methods proposed in this work is new and original; (ii) no intelligent techniques for prompt anomaly detection, fault diagnosis and precursor identification have yet been developed for, and applied to, nuclear fusion systems.

The remainder of the paper is organized as follows. In Section 2, a description of a typical SMCCC and the thermo-hydraulic code employed to simulate its behavior [35] is briefly recapped. In Section 3, the method developed for LOFA precursor identification is presented. The approach is tested in Section 4, where the main results are shown and the comparison to the results obtained in [48] is presented. Finally, conclusions are drawn in Section 5 .

\section{The Superconducting Magnet Cryogenic Cooling Circuit (SMCCC)}

Supercritical Helium (SHe) is kept in motion in the SMCCC to cool down each CSM [68]. Figure 1 shows a simplified scheme of the SMCCC circuit with its main components. At nominal operational conditions, a nominal flow $G_{0}=0.32 \mathrm{~kg} / \mathrm{s}$ in the two cryolines and a downstream pressure $p_{0}=0.42 \mathrm{MPa}[28,69]$ is ensured by a Centrifugal Pump (CP); the heat produced in the CP by the compression and in the CSM by AC losses due to pulsed operation is extracted from the SHe (in the heat exchangers HX1 and HX2, respectively) by Liquid Helium ( $\mathrm{LHe}$ ) at saturated conditions $\left(T_{\text {sat }}=4.5 \mathrm{~K}\right)$. In the SMCCC under analysis, several valves are present: the Control Valves (CV1 and CV2) are Normally Open (NO), whereas the two Safety Valves (SV1 and SV2) and the By-pass Valve (BV) are Normally Closed (NC); flow meters and pressure detectors send signals to controllers $\mathrm{C} 1$ and $\mathrm{C} 2$, respectively. Note that the SMCCC reported in Figure 1 is just a specific simplified configuration of the much more complex loops under construction in ITER, but it is relevant to develop and test the novel approach proposed here.

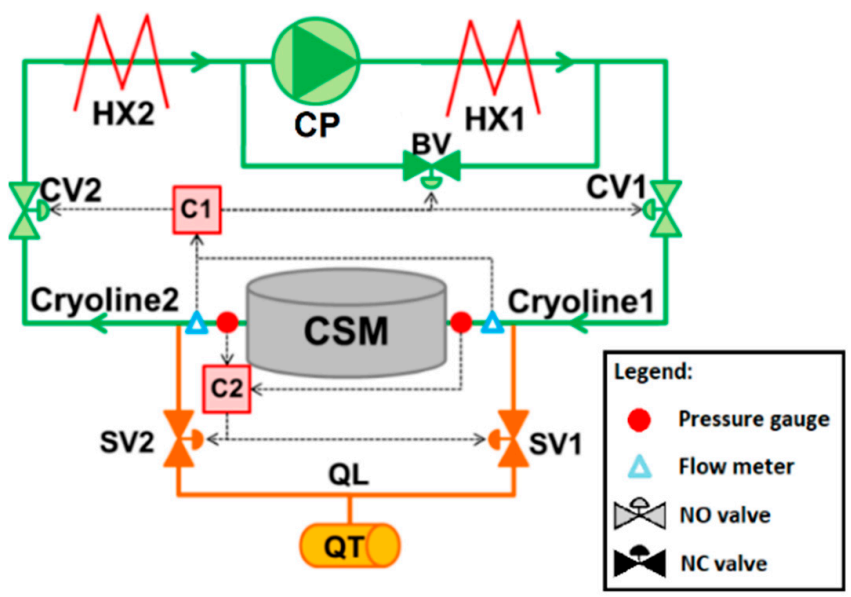

Figure 1. Simplified SMCCC.

When a LOFA occurs, and the coolant flow falls below $10 \%$ of the nominal value for more than a validation time ( $\tau_{v a l}=1 \mathrm{~s}$ in this paper) both at the CSM inlet and the CSM outlet [9]: 
- $\quad \mathrm{C} 1$ closes CV1 and CV2, opens BV preventing the CP damage and dumps the current inside the CSM in $30 \mathrm{~s}$ [70]: in this way, the SHe flows only through the by-pass line, so that the CSM is isolated and the quantity of bi-phase helium at CP upstream is reduced.

- $\quad$ C2 opens the two SVs with a PID controller when the pressure in the CSM goes beyond $p_{\text {lim }}=1.8 \mathrm{MPa}$. By so doing, $\mathrm{SHe}$ is sent to the Quench Tank $(\mathrm{QT})$, at pressure $p_{Q T}=0.35 \mathrm{MPa}$ and temperature $T_{Q T}=300 \mathrm{~K}$ [28]: thus, the pressure limit of $25 \mathrm{MPa}$ is not exceeded and the integrity of the joints adjacent to the CSM during quench is guaranteed.

The closed cooling circuit is simulated for a mission time $t_{\text {miss }}=3600 \mathrm{~s}$ (at the beginning of which the current evolution foreseen for the ITER 15 MA plasma scenario is followed) with the $4 \mathrm{C}$ code that includes [35]: (i) a 1-D thermal-hydraulic model for each channel of the CSM that is thermally coupled with the others through a $2^{1 / 2}$-D model accounting for heat diffusion phenomena in each radial section of the CSM; (ii) a 1-D compressible fluid model for pipes and HXs; (iii) a 0-D model for the mass and the energy balance in relevant points of the cooling loop (such as valves, QT, CP, etc.). The choice of the mission time $t_{\text {miss }}=3600 \mathrm{~s}$ is dictated by the length and shape of one single pulse of current in the ITER CSM, which can be divided into $Q=5$ phases:

- $\quad$ First Magnetization phase (FM): in the first $130 \mathrm{~s}$, a variation in the current from $40 \mathrm{kA}$ to $-40 \mathrm{kA}$ in $80 \mathrm{~s}$ is registered, which leads to large AC losses and eddy currents.

- $\quad$ Burning Phase (B): the current decreases to $-45.5 \mathrm{kA}$ for $386 \mathrm{~s}$.

- $\quad$ Rump Down phase (R): the current reaches $0 \mathrm{kA}$ at $975 \mathrm{~s}$.

- Dwell phase (D): no current flows in the CSM until $1490 \mathrm{~s}$ (after the heat load of the previous phases, it is necessary to cool down the CSM).

- Last Magnetization phase (M): the current returns to the initial value of $40 \mathrm{kA}$ and, after a plateau of $10 \mathrm{~s}$, the pulse starts again for other $1800 \mathrm{~s}$.

Further physical details are not reported here for brevity, since they go far beyond the methodological scope of the present work: the interested reader is referred to $[48,68]$.

For each $i$-th simulation, we monitor $Z=3$ variables $y_{i}^{k}(t)[k=1,2, \ldots, Z]$ at time $t$. Relying on the experience of some of the authors of this paper in the thermal-hydraulic modelling of superconducting magnets systems, these $Z=3$ variables are identified as (some of the most) critical for the safe functioning of the SMCCC under analysis and worth to be monitored for a prompt and accurate detection of LOFAs, which is the main objective and interest of the present study. In particular, the pressure $p_{C S M, i n}$ at the inlet of the CSM $(k=1)$ must not exceed $p_{\text {lim }}=1.8 \mathrm{MPa}$ to ensure the integrity of joints and headers in the SMCCC. Additionally, the hotspot temperature $T_{h s}$ in the CSM $(k=2)$ and the ratio $I / I_{c r}$ between the current flowing in the conductors used to wind the CSM and the critical one (at a given magnetic field, temperature and mechanical stress) $(k=3)$ must not exceed the minimum current sharing temperature during the whole scenario simulated here, i.e., $T_{\mathcal{C S}}=7.3 \mathrm{~K}$ (corresponding to the nominal evolution of strain and magnetic field) and the ratio $\left(I / I_{c r}\right)_{l i m}=0.5$ (conservatively chosen here just to keep sufficiently far from the onset of a current sharing), respectively. The last two conditions (on $T_{h s}$ and $I / I_{c r}$ ) aim at avoiding ohmic heating (due to current flowing in the copper strands) and at guaranteeing the superconductive properties in the CSM, thus preventing quench. Some considerations are in order in this respect. First, we have relied entirely on expert knowledge and on the physical considerations above for the selection of $p_{C S M, i n}$ $T_{h s}$ and $I / I_{c r}$, as they were deemed to be relevant for the early identification of the type of accident of interest to the present study, i.e., a LOFA, and of the corresponding precursors. Obviously, the analysis of a different class of accident scenarios would possibly require the selection of a different set of monitored parameters. Additionally, more advanced automatic techniques could be employed in the future for the optimal identification of the variables to be used by the detection model, e.g., feature extraction [71] and selection [72] algorithms. Second, the size of the set of monitored parameters (i.e., $Z=3$ ) has been chosen arbitrarily by the authors: on the one hand, it is small enough to make the physical 
analysis of the results easy and tractable; on the other hand, it still allows to demonstrate the performance of the proposed framework for LOFA detection on a relatively simple yet realistic multi-dimensional problem, which is the main methodological scope of the present paper. Finally, notice that the numerical values of the failure limits (discussed and motivated above), i.e., $p_{l i m}=1.8 \mathrm{MPa}, T_{\mathcal{C S}}=7.3 \mathrm{~K}$ and $\left(I / I_{c r}\right)_{l i m}=0.5$, do not have any impact on the algorithmic structure, generalization properties and applicability of the overall methodological framework for LOFA detection and precursor identification here proposed.

The thresholds for $p_{C S M, i n}, T_{h s}$ and $I / I_{c r}$ may be exceeded due to failures of the mechanical components (CP, CV1, CV2, BV, SV1 and SV2) that are characterized by different magnitudes and timings [28]. In particular [48]:

- The rotational speed of the CP may decrease and lead to reducing the mass flow rate at (i) $75 \%$, (ii) $50 \%$, (iii) $25 \%$ or (iv) $0 \%$ of the nominal value: i.e., for simplicity, five (equally spaced) levels of performance are selected between $0 \%$ and $100 \%$ for CP.

- $\quad \mathrm{NO}$ valves (CV1 and CV2) may fail in three different ways: (i) stuck opened at the nominal position; (ii) partially closed with a flow area at $50 \%$ of the nominal one; (iii) completely closed. For simplicity, three different (equally spaced) functioning states are chosen between $0 \%$ and $100 \%$ for CV1 and CV2.

- $\quad \mathrm{NC}$ valves (BV, SV1 and SV2) may fail in three different ways: (i) stuck closed at the nominal position; (ii) partially opened with a flow area at $50 \%$ of the nominal one; (iii) completely opened. Again, for simplicity, three different (equally spaced) functioning states are chosen between $0 \%$ and $100 \%$ for BV, SV1 and SV2.

Notice that, as before, the different states of (full, degraded and failed) performance of the mechanical components have been chosen arbitrarily by the authors: on the one hand, the number of functioning states for each component is small enough to make the physical analysis of the precursor identification results manageable and relatively easy to describe; on the other hand, the random transitions of the components between such performance levels at different times generate a large number of states for the overall system: this allows to demonstrate the effectiveness of the proposed framework for LOFA precursor identification on a relatively simple, but realistic multi-state dynamic problem, which is the main methodological scope of the present work. In addition, notice that the (arbitrary) fineness of the discretization of the performance of the (input) components only determines the overall number of available states, which the system can visit during its mission time. However, it does not have any impact on the algorithmic structure, generalization properties and applicability of the methodological framework for LOFA detection and precursor identification here proposed.

\section{LOFA Precursors Identification: The Proposed Framework}

We propose a three-step method for the "online" characterization of newly developing scenarios relying on the analysis of the $Z=3$ time-varying monitored signals [33,34]: (i) creation of a "database" of simulated accidental scenario (Section 3.1); (ii) clustering of the scenarios according to a measure of similarity, in order to characterize the "prototypical" evolutions of the system toward critical conditions (i.e., LOFA), together with the corresponding precursors (i.e., component failures) (Section 3.2); (iii) comparison of a new scenario with those belonging to the created database, in order to infer the possible component failures responsible for the signal evolution (i.e., of the system's critical condition) (Section 3.3). The entire framework is summarized and sketched in Figure 2 for the sake of clarity. 


\section{Accident scenarios simulated by the} original $4 \mathrm{C}$ model code

Step 1: Creation of a "database" of simulated accidental scenarios (Section 3.1)

a) Adaptive Kriging - Monte Carlo Sampling (AK-MCS) (Section 3.1.1)

b) Proper Orthogonal Decomposition (POD)-based Kriging metamodels (Section 3.1.2)

Large number of (new) time-varying signals (safety-critical variables) enriching the database of accidental scenarios

Step 2: Prototypical transients and components failure modes identification (Section 3.2)

a) Spectral Clustering (SC) embedding the Fuzzy C-Means (FCM) (Section 3.2.1)

b) Post-processing of the clusters (Section 3.2.2)

- Clusters of scenarios, grouped according to similar functional behavior - Prototypical LOFA occurrence times and components failure modes

Step 3: On-line Supervised Spectral Clustering (OSSC) (Section 3.3)

LOFA occurrence times and precursors for new, developing transients

Figure 2. Flowchart of the proposed framework for LOFA precursor identification.

\subsection{Step 1: Creation of a "Database" of Simulated Accidental Scenarios}

We represent each i-th accident scenario of the "database" by a sequence of events, encoded by a vector of $M=12$ elements $\boldsymbol{X}_{i}=\left[m_{C P}, \tau_{C P}, m_{C V 1}, \tau_{C V 1}, m_{C V 2}, \tau_{C V 2}, m_{B V}, \tau_{B V}\right.$, $\left.m_{S V 1}, \tau_{S V 1}, m_{S V 2}, \tau_{S V 2}\right]$ and generated by Monte Carlo Sampling (MCS): for each component, the magnitude $(m)$ of the failure and the time $(\tau)$ at which the failure occurs are listed [28] (it is straightforward to notice that if the number of mechanical components considered changes (e.g., because the analyst wants to include more valves, more pumps or additional pipelines), this means that we are analyzing a different physical system and correspondingly a different thermal-hydraulic model (with a different number of inputs). Thus, a different metamodel should be coherently constructed and trained).

The magnitude $(m)$ is assumed as follows:

- $\quad$ Discrete values between 0 and 4 are used to indicate the magnitude of the CP failure. If the component has not failed, $m_{C P}=0$ in the corresponding state vector. Instead, $m_{C P}$ values equal to $1,2,3$ or 4 correspond to states of reduction of the mass flow rate of $75 \%, 50 \%, 25 \%$ or $0 \%$ of the nominal value, respectively, due to a decrease of its rotational speed. 
- Discrete values between 0 and 3 are employed to characterize the magnitude of NO valves (CV1 and CV2) failures. If the component works correctly, $m=0$. Instead, if the valve remains stuck opened, partially closed with a reduction of the flow section area of $50 \%$ or completely closed, then $m$ is set to 1,2 or 3 , respectively.

- Discrete values between 0 and 3 are used to indicate the magnitude of NC valves (BV, SV1 and SV2) failures, too. When the component has not failed, $m=0$. Otherwise, when the valve remains stuck closed, partially opened with the flow section area at $50 \%$ of the one completely opened or completely opened, then $m$ is set to 1,2 or 3, respectively.

The failure time $(\tau)$ is a discrete value between $0 \mathrm{~s}$ and $1800 \mathrm{~s}$ (the discretization step is chosen equal to $0.01 \mathrm{~s}$ ). Notice that such time interval has been selected because $1800 \mathrm{~s}$ is the length of a single pulse of current in the ITER CSM. In case a different mission time, $\tau_{\text {miss }}$ is of interest to the analysis, the failure time $(\tau)$ values will be straightforwardly sampled within the range $\left[0, \tau_{\text {miss }}\right] \mathrm{s}$. If a value of 0 is indicated for $\tau$, this means that the component has not failed.

For instance, if the vector $\boldsymbol{x}_{i}$ is equal to [2,60, 2, 1785, 1, 689, 0, 0, 1, 856, 0, 0], the scenario to be simulated by the $4 \mathrm{C}$ entails: failure of the $\mathrm{CP}$ at $60 \mathrm{~s}$ with the flow at $50 \%$ of the nominal value; valves CV2 and SV1 stuck at their nominal position at $689 \mathrm{~s}$ and $856 \mathrm{~s}$, respectively; and partial closing of CV1 at 1785 s, whereas the BV and SV2 work correctly during the entire transient

Once vector $\boldsymbol{X}_{i}$ is sampled, it is sent an an input to the $4 \mathrm{C}$ code, which outputs the three critical variables $y_{i}^{k}(t)[k=1,2,3]$ and the mass flow rates, $G_{C S M, \text { in }}(t)$ and $G_{C S M, o u t}(t)$, at the inlet and at the outlet of the CSM, respectively. When $G_{C S M, i n}(t)<0.032 \mathrm{~kg} / \mathrm{s}$ and $G_{C S M, o u t}(t)<0.032 \mathrm{~kg} / \mathrm{s}$ for more than the validation time $\left(\tau_{\text {val }}=1 \mathrm{~s}\right)$, a LOFA takes place and is detected by controller $\mathrm{C} 1$ [31]; the LOFA detection time is indicated as $t_{L O F A, C 1, i}$.

Notice that each simulation of the system transient behavior by the $4 \mathrm{C}$ code requires on average two days on an Intel Core i3-7100 3.9 GHz 3 MB Cache. Thus, in this phase, fastrunning Kriging metamodels are used to reduce the computational burden associated with the creation of the accident scenario "database". This is done in two sub-steps. First, the Adaptive Kriging-Monte Carlo Sampling (AK-MCS) method is adopted to progressively refine the accuracy of the metamodels in reproducing the critical system configurations of our interest, i.e., the LOFA conditions [38-40] (Section 3.1.1). Based on the system statespace exploration performed by the AK-MCS, Proper Orthogonal Decomposition (POD)based Kriging metamodels [42,43] are then constructed to quickly simulate a large number of (new) time-varying signals without resorting to the original long-running $4 \mathrm{C}$ code: this allows enriching the database of accidental scenarios at a negligible computational cost (Section 3.1.2).

\subsubsection{Adaptive Kriging-Monte Carlo Sampling (AK-MCS) Method}

The Adaptive Kriging-Monte Carlo Sampling (AK-MCS) algorithm [37,39,40] is an advanced metamodel-based random sampling method here used to generate and include in the database "interesting" scenarios lying in proximity of system failure configurations (i.e., LOFA conditions) intelligently and adaptively. In such scenarios, the maximum values of the critical variables $\boldsymbol{y}_{i}=\left[\left(y_{i}^{1}(t)\right)_{\max ^{\prime}}\left(y_{i}^{2}(t)\right)_{\max }\left(y_{i}^{3}(t)\right)_{\max }=\left[\mathcal{Y}_{i}^{1}, \mathcal{Y}_{i}^{2}, \mathcal{Y}_{i}^{3}\right]\right.$ lie in proximity of the critical safety thresholds $\boldsymbol{y}_{t h r}=\left[\mathcal{Y}_{t h r}^{1}, \mathcal{Y}_{t h r}^{2}, \mathcal{Y}_{t h r}^{3}\right]=\left[p_{l i m}=1.8 \mathrm{MPa}\right.$, $\left.\mathrm{T}_{\mathcal{~} S}=7.3 \mathrm{~K},\left(I / I_{c r}\right)_{l i m}=0.5\right]$. This criterion is used to drive the simulations preferably towards "critical" scenarios and system configurations, without wasting computational time in the exploration of safe (not interesting) areas of the system state-space.

The adaptive procedure, shown in the flow chart of Figure 3 and detailed below, is applied to each $k$-th output (i.e., safety-critical) variable of interest [40]: 
- $\quad$ Step (0) An initial Design Of Experiment (DOE) $(\overline{\boldsymbol{x}}, \overline{\boldsymbol{y}})$ (or training set), i.e., a set of examples/realizations $\left(\boldsymbol{x}_{i}, \boldsymbol{y}_{i}\right)\left(i=1, \ldots, N_{\text {train }}\right)$ of the input-output relationship underlying the original $4 \mathrm{C}$ code, is first created, including all the inputs $\overline{\boldsymbol{X}}=\left\{\boldsymbol{x}_{1}, \boldsymbol{X}_{2}, \ldots, \boldsymbol{x}_{N_{\text {train }}}\right\}$ and the corresponding simulated outputs $\overline{\boldsymbol{y}}=\left\{\boldsymbol{y}_{1}, \boldsymbol{y}_{2}, \ldots\right.$.

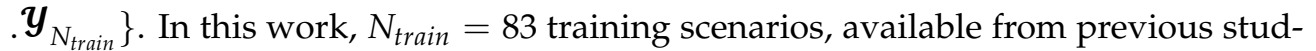
ies [26], are employed as the initial DOE.

- $\quad$ Step (1) Set $N_{\text {krig }}=N_{\text {train }}$. The DOE $(\overline{\boldsymbol{x}}, \overline{\boldsymbol{y}})$, set of elements $\left(\boldsymbol{x}_{i}, \boldsymbol{y}_{i}\right)(i=1, \ldots$, $N_{\text {krig }}$ ), is employed to train one Kriging metamodel $\mathcal{M M}^{k}$ for each output $k=1,2,3$. Such surrogate models are adopted for their capability of approximating and reproducing complex nonlinear functions and of providing an estimate of the uncertainty associated with their predictions on new, unknown inputs [36,41,73]. The parameters of these metamodels are "tuned" according to the guidelines given in ref. [39] (see Appendix A for details).

- $\quad$ Step (2) New $N_{M C S}=100,000$ (input) scenarios $\overline{\boldsymbol{x}}_{M C S}=\left\{\boldsymbol{x}_{1}, \boldsymbol{x}_{2}, \ldots, \boldsymbol{x}_{N_{M C S}}\right\}$ (different from the $N_{\text {krig }}$ ones) are generated by standard Monte Carlo Sampling (MCS) and the corresponding Kriging predictions (i.e., mean values) will be $\hat{\boldsymbol{Y}}_{M C S}\left(\overline{\boldsymbol{x}}_{M C S}\right)=$ $\mathcal{M M}^{k}\left(\overline{\boldsymbol{x}}_{M C S}\right)=\boldsymbol{\mu}_{\hat{\Upsilon}^{k}}\left(\overline{\boldsymbol{x}}_{M C S}\right)=\left\{\mathcal{M M}^{k}\left(\boldsymbol{x}_{1}\right), \ldots, \mathcal{M M}^{k}\left(\boldsymbol{X}_{N_{M C S}}\right)\right\}=\left\{\mu_{\hat{\gamma}^{k}}\left(\boldsymbol{x}_{1}\right)\right.$, $\left.\ldots, \mu_{\hat{\Upsilon}^{k}}\left(\boldsymbol{X}_{N_{M C S}}\right)\right\}$ and variances $\sigma_{\hat{\gamma}^{k}}^{2}\left(\overline{\boldsymbol{X}}_{M C S}\right)=\left\{\sigma_{\hat{\Upsilon}^{k}}^{2}\left(\boldsymbol{X}_{1}\right), \ldots, \sigma_{\hat{\Upsilon}^{k}}^{2}\left(\boldsymbol{X}_{N_{M C S}}\right)\right\}$ are obtained. Notice that these evaluations (i.e., the approximations of the code outputs) are computed by the metamodel at a reduced computational cost (i.e., of the order of few seconds).

- Step (3) The $N_{M C S}$ scenarios are analyzed to find those ones lying "in proximity" of the system failure region. To this aim, the learning function $\mathcal{U}^{k}(\boldsymbol{X})$ shown in Equation (1) is used:

$$
\mathcal{U}^{k}(\boldsymbol{X})=\frac{\left|\mu_{\hat{\Upsilon}^{k}}(\boldsymbol{X})-\mathcal{Y}_{t h r}^{k}\right|}{\sigma_{\hat{\Upsilon}^{k}}(\boldsymbol{X})}
$$

A low value of the learning function $\mathcal{U}^{k}(\boldsymbol{X})$ (1) means that: (i) the system configuration $\boldsymbol{X}$ is close to the failure region (i.e., the system response $\mu_{\hat{\gamma}^{k}}(\boldsymbol{X})$ is close to the failure threshold $\mathcal{Y}_{t h r}^{k}$ ); and/or (ii) the uncertainty $\sigma_{\hat{\gamma}^{k}}(\boldsymbol{X})$ associated with the Kriging prediction $\mu_{\hat{\gamma^{k}}}(\boldsymbol{X})$ is high (this is typically due to the scarcity of DOE training examples around $\boldsymbol{X}$, which prevents the metamodel from producing precise estimates: in this view, adding new examples in that critical area would improve the accuracy and precision of the metamodel).

Let us define $S_{\mathbb{M}}^{(k)}$ as the region containing a subset of the $N_{M C S}$ scenarios characterized by "low" learning function values for output $Y^{k}(\boldsymbol{X})$ :

$$
S_{\mathbb{M}}^{(k)} \equiv\left\{\boldsymbol{X}: \mathcal{U}^{k}(\boldsymbol{X})<\varepsilon\right\},
$$

where $\varepsilon$ is a properly selected "confidence" coefficient. Region $S_{\mathbb{M}}^{(k)}$ includes those system configurations $\boldsymbol{X}$, for which the critical variable $Y^{k}(\boldsymbol{X})$ (estimated by $\mu_{\hat{\gamma}^{k}}(\boldsymbol{X})$ ) is included between $\mathcal{Y}_{t h r}^{k}-\varepsilon \times \sigma_{\hat{Y}^{k}}(\boldsymbol{X})$ and $\mathcal{Y}_{t h r}^{k}+\varepsilon \times \sigma_{\hat{\Upsilon}^{k}}$ (i.e., it lies "around" the failure threshold with a given confidence). In other words, the values $\mathcal{Y}_{t h r}^{k} \pm \varepsilon \times \sigma_{\hat{Y}^{k}}(\boldsymbol{X})$ represent lower and upper boundaries of the (failure) limit state surface including the prediction uncertainty of the Kriging metamodel. In this view, $\varepsilon$ sets the confidence level: by way of example, for a two-sided $\alpha=95 \%$ Confidence Interval (CI) $\varepsilon=\Phi^{-1}\left(\frac{1+\alpha}{2}\right)=\Phi^{-1}(0.975)=1.96$ (where $\Phi^{-1}(\cdot)$ is the inverse cumulative distribution function of the Normal distribution). By way of example, the lower boundary estimates the limit state surface under the assumption that the real value of every sample $\boldsymbol{X}$ is $\mathcal{Y}_{t h r}^{k}-\varepsilon \times \sigma_{\hat{\Upsilon}^{k}}(\boldsymbol{X})$ instead of the mean value $\mu_{\hat{\gamma}^{k}}(\boldsymbol{X})$ [38]. 


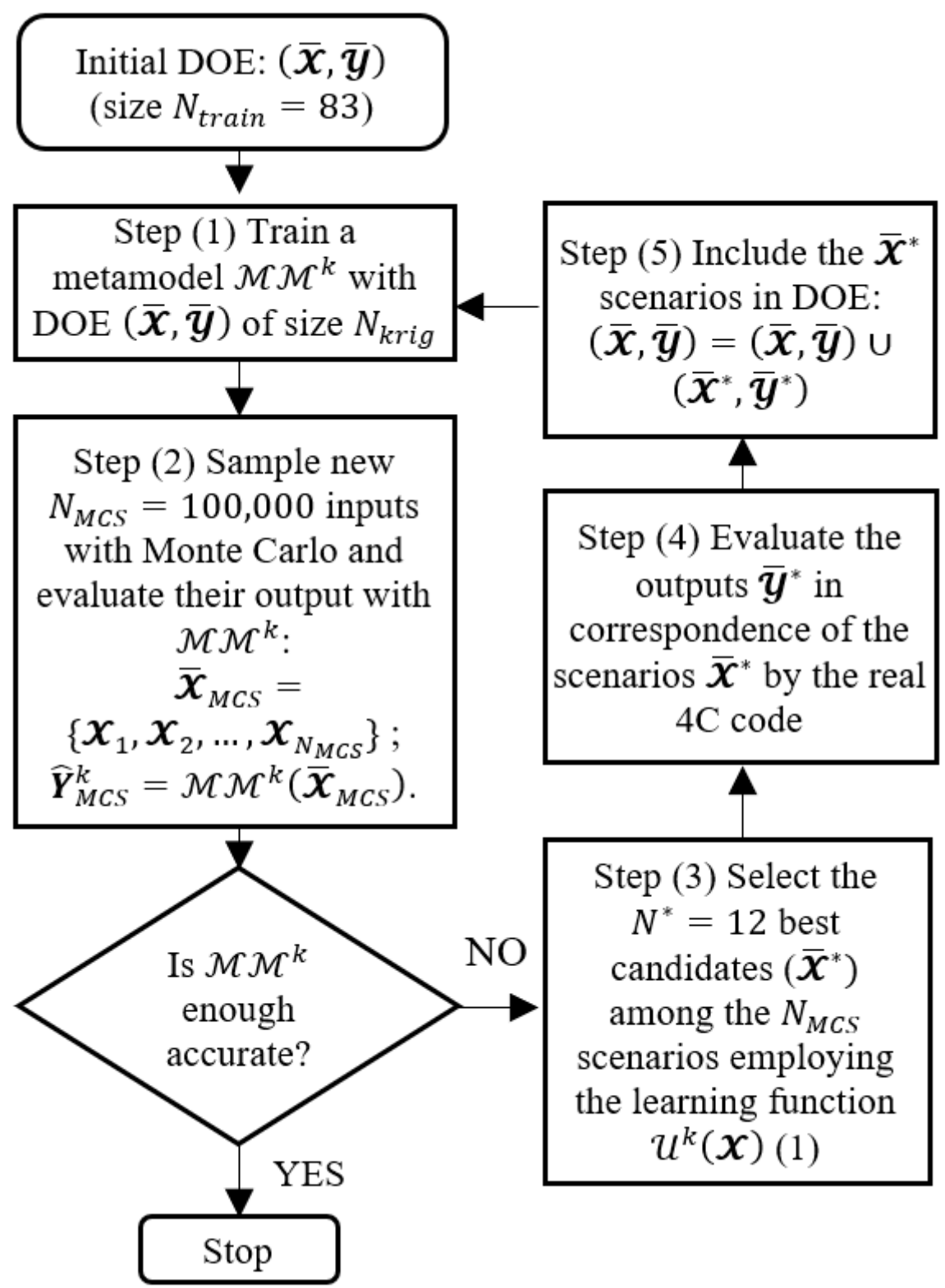

Figure 3. Flowchart of the AK-MCS method.

Once $S_{\mathbb{M}}^{(k)}$ is identified, a set of $N^{*}$ input configurations, "evenly distributed" in the confidence region $S_{\mathbb{M}}^{(k)}$, is selected to be added to the current DOE (these new configurations are used to update and enrich the current DOE with "interesting" scenarios that will likely lead the system to failure). It is shown in refs. [37-40] that optimal performances of the algorithm are typically obtained for $N^{*}$ lying between $M$ and $2 M$. Coherently, in this work we set $N^{*}=M=12$ in order to obtain satisfactory performances at an affordable computational cost (i.e., reducing the number of additional simulations by the detailed $4 \mathrm{C}$ code as much as possible).

- Step (4) The identified $N^{*}=12$ input configurations $\overline{\boldsymbol{X}}^{*}$ are simulated by the original $4 \mathrm{C}$ code, finding the corresponding outputs $\overline{\boldsymbol{y}}^{*}$. 
- $\quad$ Step (5) The new (input-output) training examples $\left(\overline{\boldsymbol{x}}^{*}, \overline{\boldsymbol{y}}^{*}\right)$ are added to the current DOE $(\overline{\boldsymbol{x}}, \overline{\boldsymbol{y}})=(\overline{\boldsymbol{x}}, \overline{\boldsymbol{y}}) \cup\left(\overline{\boldsymbol{x}}^{*}, \overline{\boldsymbol{y}}^{*}\right)$, whose size becomes $N_{k r i g}=N_{k r i g}+N^{*}$.

- Steps (1)-(5) are repeated until a desired level of accuracy of the metamodel $\mathcal{M M}^{k}$ is reached.

\subsubsection{Proper Orthogonal Decomposition (POD)-Based Kriging Metamodels}

In order to simulate a large number of new (time-varying) outputs $y^{k}(t)$ at a reduced computational cost (i.e., without resorting to the $4 \mathrm{C}$ code), an algorithm (still based on Kriging metamodels) is adopted. These new (approximate) transients will be employed for further enrichment of the available database of accident scenarios.

Each $i$-th scenario $\left(i=1, \ldots, N_{\text {krig }}\right)$ generated in the previous step by the $4 \mathrm{C}$ code simulations (Section 3.1.1) is decomposed by resorting to POD with truncation at the $H_{k}$-th basis [20]:

$$
y_{i}^{k}(t)=\sum_{h=1}^{H_{k}} a_{i h}^{k}\left(\boldsymbol{x}_{i}\right) \times \varphi_{h}^{k}(t)
$$

where $\varphi_{h}^{k}(t)$ (depending only on time $t$ ) is the orthogonal basis function of the $k$-th variable for the $h$-th base valid for all the $N_{k r i g}$ scenarios, and $a_{i h}^{k}\left(\boldsymbol{X}_{i}\right)$ (depending only on the input configuration $\boldsymbol{X}_{i}$ ) is its coefficient corresponding to the $i$-th scenario.

Each orthogonal function $\varphi_{h}^{k}(t)$ is characterized by the property shown in Equation (4):

$$
\int_{t=0 s}^{t=t_{m i s s}} \varphi_{h_{1}}^{k}(t) \times \varphi_{h_{2}}^{k}(t) \times d t=\delta_{h_{1} h_{2}}=\left\{\begin{array}{l}
0 \text { if } h_{1}=h_{2} \\
1 \text { if } h_{1} \neq h_{2}
\end{array}\right.
$$

Thanks to this property, each coefficient $a_{i h}^{k}\left(i=1, \ldots, N_{k r i g} ; h=1, \ldots, H_{k}\right)$ can be easily estimated by Equation (5):

$$
a_{i h}^{k}=\int_{t=0 s}^{t=t_{m i s s}} y_{i}^{k}(t) \times \varphi_{h}^{k}(t) \times d t
$$

In order to employ this strategy for the simulation of new scenarios, the following procedure is performed for each $k$-th critical output variable $y^{k}(t)(k=1,2,3)$ :

- Step (1): The database of $N_{\text {krig }}$ scenarios is employed to build a matrix $\stackrel{\bar{Y}}{Y}\left[N_{\text {krig }}, L\right]$, containing the value $y_{i l}^{k}$ of the $k$-th variable of the $i$-th training scenario at the $l$-th time step.

- $\quad$ Step (2): The matrix $\stackrel{=}{Y}$ is decomposed by Singular Value Decomposition (SVD) [33] as in Equation (6):

$$
\stackrel{=}{Y}=\stackrel{\bar{\Psi}}{\Psi}^{k} \times \stackrel{\bar{\Lambda}}{\Lambda}^{k} \times \overline{\bar{\Phi}}^{k}
$$

where $\stackrel{\stackrel{{ }^{\Psi}}{\Psi}}{k}\left[N_{\text {krig }}, N_{\text {krig }}\right]$ and $\stackrel{{ }^{\Phi}}{\Phi}[L, L]$ are matrices containing in their columns leftsingular vectors and right-singular vectors, respectively, whereas ${ }^{k}\left[N_{k r i g}, L\right]$ is a diagonal matrix containing the nonnegative $\Lambda_{h}^{k}$ singular values in decreasing order.

- Step (3): The best number $H_{k}$ of bases retained is identified using the singular values $\Lambda_{h}^{k}\left(h=1, \ldots, N_{k r i g}\right)$ by Equation (7):

$$
\Gamma_{h}^{k}=\frac{\sum_{j=1}^{h} \Lambda_{j}^{k}}{\sum_{h=1}^{N_{k r i g}} \Lambda_{h}^{k}}
$$


where $\Gamma_{h}^{k}$ represents the percentage of "variability" (i.e., variance) of the real $N_{k r i g}$ transients that is "explained" by the POD decomposition truncated at $h$-th basis. In this work, $H_{k}$ is the number of (ordered) bases for which $\Gamma_{h}^{k}$ reaches a value of 0.99 (i.e., for which the POD decomposition can explain the $99 \%$ of the total variance of the real transients). Matrix $\overline{\bar{\Phi}}^{k}\left[L, H_{k}\right]$ is then truncated at the $H_{k}$-th column: its generic element $\varphi_{l h}^{k}$ at the $l$-th row and $h$-th column corresponds to the value of the $h$-th orthogonal basis $\varphi_{h}^{k}(t)$ at $l$-th time step for output $k$.

- $\quad$ Step (4): Matrix $\overline{\bar{A}}^{k}\left[N_{k r i g}, H_{k}\right]$, containing coefficients $a_{i h}^{k}$ in the $i$-th row and the $h$-th column, is calculated as in Equation (8):

$$
\overline{\bar{A}}^{k}=\overline{\bar{Y}}^{k} \times \overline{\bar{\Phi}}^{\mathrm{\Phi}}
$$

where $\overline{\bar{\Phi}}^{k^{T}}\left[H_{k}, L\right]$ is the transposal of $\overline{\bar{\Phi}}^{k}$. This equation represents a discretized form of Equation (5).

- Step (5): We build a Kriging metamodel $\mathcal{M M}_{h}^{k}$ for each $h$-th base and $k$-th critical variable. The training set (DOE) is constituted by the inputs $\bar{x}=\left\{\boldsymbol{x}_{1}, \boldsymbol{x}_{2}, \ldots, \boldsymbol{x}_{\mathrm{N}_{\text {krig }}}\right\}$ and the corresponding basis coefficients $\boldsymbol{a}_{h}^{k}=\left\{a_{1 h^{\prime}}^{k} a_{2 h^{\prime}}^{k}, \ldots, a_{N_{k r i g} h}^{k}\right\}$ as outputs. In this way, for a new input configuration $\boldsymbol{X}$, the coefficients of the POD decomposition can be estimated as $\hat{a}_{h}^{k}(\boldsymbol{x})=\mathcal{M} \mathcal{M}_{h}^{k}(\boldsymbol{x})$.

- $\quad$ Step (6): We provide a discretized approximation to the time-varying output $y_{j}^{k}(t)$ for a new $j$-th scenario at the $l$-th time step $(l=1, \ldots, L)$ as in Equation (9):

$$
\widetilde{y}_{j l}^{k}=\sum_{h=1}^{H_{k}} \hat{a}_{h}^{k}\left(\boldsymbol{x}_{j}\right) \times \varphi_{l h}^{k}
$$

where $\widetilde{y}_{j l}^{k}$ is the estimate of $y_{j l}^{k}$, resulting from the metamodel-based POD decomposition.

In this way, by resorting to multiple Kriging metamodels instead of the $4 \mathrm{C}$ code, the computational time per simulation is sharply reduced.

We employ the same approach to approximate $G_{\max , j}(t)$, i.e., the maximum between the helium mass flow rates at the inlet and outlet of the CSM, (see Equation (10)), which is here used to estimate $t_{L O F A, C 1, j}$ (i.e., of the time when a LOFA occurs and is detected by C1) for the $j$-th scenario:

$$
G_{\max }(t)=\max \left(G_{C S M, \text { in }}(t), G_{C S M, o u t}(t)\right)
$$

We indicate the approximation of $G_{\max , j}(t)$ by POD-based Kriging metamodels for a generic $j$-th scenario at $l$-th time step as $\widetilde{G}_{m a x, l j}$. Then, $\widetilde{t}_{L O F A, C 1, j}$ (i.e., the POD metamodelbased approximation of $\left.t_{L O F A, C 1, j}\right)$ is the time where $\widetilde{G}_{m a x, l j}<0.032 \mathrm{~kg} / \mathrm{s}$ for more than the validation time $\tau_{v a l}=1 \mathrm{~s}$ (thus, mimicking the operation of the controller $\mathrm{C} 1$ ). The total number of scenarios simulated by the POD-based metamodels (and included in the "database") is indicated as $N_{P O D}$ (notice that typically $N_{P O D} \gg N_{k r i g}$, due to the fast-running property of the Kriging metamodels with respect to the $4 \mathrm{C}$ code).

For the sake of clarity, a pictorial representation of the conceptual steps (1)-(6) above, undertaken to construct the POD-based Kriging metamodels, is given in Figure 4. 


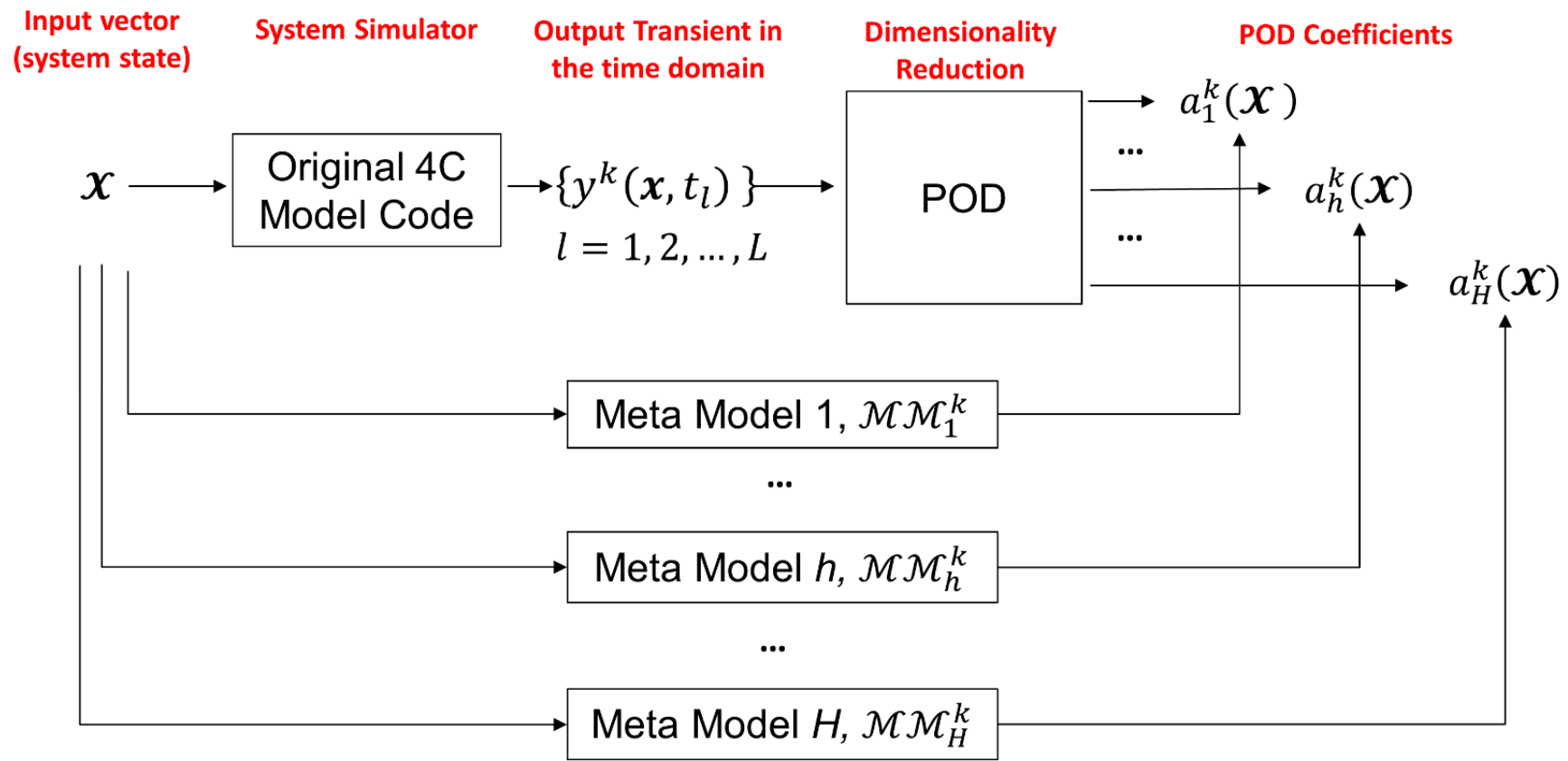

Figure 4. Conceptual steps undertaken to construct the POD-based Kriging metamodels.

\subsection{Step 2: Prototypical Transients and Components Failure Modes Identification}

From the previous step, a database is available constituted by $N_{k r i g}$ and $N_{P O D}$ scenarios simulated with the $4 \mathrm{C}$ code and with POD-based Kriging metamodels, respectively. Based on Spectral Clustering (SC), we classify the $N_{\text {data }}=N_{\text {krig }}+N_{P O D}$ transients in C clusters $[28,44,74,75]$. Then, we post-process each $c$-th cluster to extract its main features, in terms of prototypical time evolutions towards failure and of the corresponding component failures (i.e., the accident precursors) $[45,46,76]$.

\subsubsection{Spectral Clustering (SC) Embedding the Fuzzy C-Means (FCM)}

Spectral Clustering (SC) allows to classify $N_{\text {data }}$ objects into $C$ clusters through a similarity measure (namely, $w$ ) between them (see Appendix B). We compute all the similarity values using the three critical variables $y_{i}^{k}(t)[k=1,2,3]$ (constituted by $L$ time steps) and we collected them in the similarity matrix $\stackrel{\bar{W}}{W}\left[N_{\text {data }}, N_{\text {data }}\right]$, from which the Normalized Laplacian matrix $\overline{\bar{L}}_{s y m}$ is calculated. We extract the features needed to classify the $N_{\text {data }}$ objects from $\overline{\bar{L}}_{\text {sym }}$ and we feed them to the Fuzzy C-Means (FCM) code.

The algorithm outputs two matrices: (i) a matrix $\overline{\mathcal{A}}[C, C]$ containing the eigenspace coordinates of the center of the $c$-th cluster in each $c$-th row (in this notation, $\overline{\mathcal{A}}_{c}=$ $\left[\mathcal{A}_{c 1}, \mathcal{A}_{c 2}, \ldots, \mathcal{A}_{c C}\right]$ is the vector containing such coordinates); (ii) a matrix $\overline{\bar{M}}\left[C, N_{\text {data }}\right]$ whose generic element is the $M_{c i}$ membership degree of the $i$-th transient of the database respect to the $c$-th cluster: the $i$-th transient is said to belong to the cluster with the membership exceeding a certain limit (in this paper, $M_{\text {lim }}=0.7$ ).

3.2.2. Post-Processing of the Clusters to Identify the LOFA Occurrence Time and the Components Failure Modes

The $N_{\text {data,c }}<N_{\text {data }}$ scenarios belonging to the $c$-th cluster are post-processed to evaluate the following quantities: $P_{L O F A, l}(c)$, which quantifies the probability that a LOFA has occurred at the $l$-th time step in a system state belonging to cluster $c$; and $P_{F A I L, l}(e \mid c)$, which describes the probability that component $e$ has failed at the $l$-th time step, given that 
a LOFA has occurred due to a system configuration of cluster $c$. The above-mentioned probabilities are quantified as in Equations (11) and (12), respectively:

$$
\begin{aligned}
& P_{\text {LOFA }, l}(c)=\frac{\sum_{i=1}^{N_{\text {data, }}} \Theta\left(t_{L O F A, C 1, i} \epsilon\left[t_{o}, t_{o+1}\right]\right)}{N_{\text {data }, c}} \\
& P_{F A I L, l}(e \mid c)=\frac{\sum_{i=1}^{N_{\text {data, }}} \Theta\left(\tau_{e, i} \in\left[t_{o}, t_{o+1}\right] \wedge m_{e, i} \neq 0\right)}{N_{\text {data }, c}} \\
& \text { with } \Theta(x)=\left\{\begin{array}{l}
0 \text { if } x \text { false } \\
1 \text { if } x \text { true }
\end{array} .\right.
\end{aligned}
$$

Here, $\left[t_{o}, t_{o+1}\right]$ is the $o$-th time interval, which the $l$-th time step belongs; and $\Theta(x)$ is the Heaviside function (Equation (13)) used to count the events of interest during that interval, i.e., the number of LOFA occurrences in Equation (11) and the $e$-th component failures $\left(m_{e} \neq 0\right)$ in Equation (12). Notice that the length of the $o$-th interval $\left[t_{o}, t_{o+1}\right]$ is here set to $300 \mathrm{~s}$, as a satisfactory compromise between analysis resolution and computational tractability (the intervals should be large enough to count a statistically meaningful number of samples). Quantities (11) and (12) thus represent a discretization (with steps of $300 \mathrm{~s}$ ) of the time-dependent probabilities described above.

\subsection{Step 3: Timely LOFA Precursors Identification by On-Line Supervised Spectral Clustering (OSSC)}

A novel Online Supervised Spectral Clustering (OSSC) method is trained with the available $N_{\text {data }}$ scenarios to timely identify LOFA precursors during the development of a new $j$-th accident scenario, different from the training ones. The $j$-th scenarios are characterized by the monitored critical variables $y_{j}^{k}(t)(k=1,2, \ldots, Z=3)$ and compared to those of the database $y_{i}^{k}(t)\left(i=1,2, \ldots, N_{\text {data }}\right)$ at each $l$-th time step. The algorithm proceeds each $j$-th scenario (the flowchart is sketched in Figure 5 ) as follows:

- $\quad$ Step (1): Record the $k$-th trajectory $y_{j}^{k}(t)$ every $\Delta t=0.01 \mathrm{~s}$ from $0 \mathrm{~s}$ to $3600 \mathrm{~s}$, obtaining values $y_{j l}^{k}(l=1,2, \ldots, L)$ at each $l$-th time step. Thus, store $L=360,001$ points for each $k$-th variable.

- $\quad$ Step (2): As suggested by ref. [77], normalize the value $y_{j l}^{k}\left(l=1,2, \ldots, L ; k=1, \ldots, N_{k}\right)$ in the range $[0.2,0.8]$ as in Equation (14) and save it as $y_{n, j l}^{k}$ (see also Appendix B):

$$
y_{n, j l}^{k}=0.2+0.6 \times \frac{y_{j l}^{k}-\min \left(\overline{\bar{Y}}^{k}\right)}{\max \left(\overline{\bar{Y}}^{k}\right)-\min \left(\overline{\bar{Y}}^{k}\right)}
$$

where $\overline{\bar{Y}}^{k}$ is the matrix containing values $y_{i l}^{k}\left(i=1, \ldots, N_{\text {data }}\right)$ for the $k$-th trajectory.

- Step (3): Evaluate the Euclidean pointwise distance $\delta_{l, j i}$ between the $j$-th new scenario and the $i$-th training scenario $\left(i=1,2, \ldots, N_{\text {data }}\right)$ of the $l$-th time step $(l=1,2, \ldots, L)$, as in Equation (15):

$$
\delta_{l, j i}=\sum_{k=1}^{Z} \sum_{p=1}^{l}\left|y_{n, j p}^{k}-y_{n, i p}^{k}\right|
$$


- $\quad$ Step (4): Build the similarity vector $\bar{W}_{l, j}\left[1, N_{\text {data }}\right]$ at each $l$-th time step, whose generic element $w_{l, j i}$ is computed as in Equation (16):

$$
w_{l, j i}=e^{-F \times \delta_{l, j i}^{2}}
$$

with $F=1.7 \times 10^{-9}$ [28]. The higher $w_{l, j i}$, the higher the similarity between the $j$-th testing scenario and the $i$-th training scenario until the $l$-th time step.

- $\quad$ Step (5): Calculate the row vector $\bar{U}_{l, j}[1, C]$ by projecting $\bar{W}_{l, j}$ in the eigenspace through Equation (A19) (see Appendix $C$ for a proof). Afterward, normalize it obtaining $\bar{T}_{l, j}[1, C]$, whose generic element $t_{l, j c}$ is expressed by Equation (A22).

- Step (6): Calculate each $M_{l, c j}$ membership $(c=1, \ldots, C)$, measuring the "level" of confidence with which the $j$-th scenario at the l-th time step "belongs" to the $c$-th cluster, as in Equation (17) (produced by the FCM algorithm-see Appendix B):

$$
M_{l, c j}=\left[\sum_{\varsigma=1}^{C}\left(\frac{\left\|\bar{T}_{l, j}-\overline{\mathcal{A}}_{c}\right\|}{\left\|\bar{T}_{l, j}-\overline{\mathcal{A}}_{\varsigma}\right\|}\right)^{\frac{2}{\rho-1}}\right]^{-1}
$$

where $\overline{\mathcal{A}}_{c}[1, C](c=1,2, \ldots, C)$ contains the eigenspace coordinates of the prototypical transient of the $c$-th cluster (i.e., the cluster center) and $\rho=2$ is the fuzzy partition exponent [78].

- $\quad$ Step (7): Evaluate the difference $M_{r e l, l, c j}$ between $M_{l, c j}(c=1,2, \ldots, C)$ and $M_{l, c 0}$ (i.e., the membership to the $c$-th cluster at $l$-th time respect of a scenario at nominal conditions with no failures):

$$
M_{r e l, l, c j}=M_{l, c j}-M_{l, c 0}
$$

By so doing, the "background" contribution of a "standard" scenario is "removed" from the membership trend of the $j$-th test transient to cluster $c$.

- $\quad$ Step (8): Calculate $V_{r e l, l, c j}(c=1,2, \ldots, C)$ by Equation (19), i.e., a discrete estimator of the derivative of the membership $M_{l, c j}$ with respect to the $l$-th time:

$$
V_{r e l, l, c j}=\left\{\begin{array}{c}
0 \text { if } l=1 \\
\frac{M_{r e l, l, c j}-M_{r e l,(l-1), c j}}{\Delta t} \text { if } l \neq 1
\end{array}\right.
$$

- $\quad$ Step (9): Identify LOFA precursors as follows: Step (9a): Compare each $V_{r e l, l, c j}[c=1, \ldots, C]$ to $V_{\text {lim, } L O F A, l}$, following the pseudo code described in Figure 6. In extreme synthesis, if at least two values of $V_{r e l, l, c j}$, namely $V_{1 s t}=\max _{c}\left(V_{r e l, l, c j}\right)$ and $V_{2 n d}=\max _{c \neq c_{1 s t}}\left(V_{r e l, l, c j}\right)$, overcome the threshold $V_{\lim , L O F A, l}$ at time $l$, then indicator $P_{L O F A, l, j}$ is assigned a value (different from zero) as in Equation (20). Finally, if $P_{L O F A, l, j}$ exceeds the threshold $P_{\text {LOFA,lim, }}$, the algorithm identifies the LOFA precursors (see step $9 \mathrm{~b}$ below); otherwise, no precursor is identified.

$$
P_{\text {LOFA }, l, j}=\frac{P_{\text {LOFA }, l}\left(c_{1 s t, l}\right)+P_{\text {LOFA }, l}\left(c_{2 n d, l}\right)}{\sum_{c}^{C} P_{\text {LOFA }, l}(c)}
$$

Notice that Equation (20) indicates the probability that a LOFA has happened at time $l$, due to a system configuration of either cluster $c_{1 s t, l}=\arg \left(\max _{c}\left(V_{r e l, l, c j}\right)\right)$ or $c_{2 n d, l}=\arg \left(\max _{c \neq c_{1 s t}}\left(V_{r e l, l, c j}\right)\right)$. 


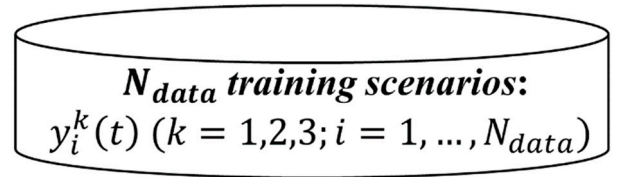

\section{On-line Cluster assignation}

Step 1: Record $y_{j l}^{k}$ from $y_{j}^{k}(t)$ and $y_{i l}^{k}$ from $y_{i}^{k}(t)(l=1, \ldots, L)$

Step 2: Calculate $y_{n, j l}^{k}$ and $y_{n, i l}^{k}$ by normalizing $y_{j l}^{k}$ and $y_{i l}^{k}$, respectively (Equation (14))

Step 3: Calculate the Euclidean pointwise distance $\delta_{l, j i}$ between the $j$-th scenario and each $i$-th training scenario $\left(i=1, \ldots, N_{\text {data }}\right)$ at $l$ th time step (Equation (15))

Step 4: Build the similarity vector $\bar{W}_{l, j}\left[1, N_{d a t a}\right]$ (Equation (16))

Step 5: Calculate $\bar{T}_{l, j}[1, C]$ (Equation (A.22)) by normalizing $\bar{U}_{l, j}[1, C]$ (i.e., the projection of the similarity vector $\bar{W}_{l, j}$ in the eigenspace - Equation (A.19))

Step 6: Calculate $M_{l, c j}(c=1, \ldots, C)$ (Equation (17))

Step $7 \&$ 8: Calculate $M_{r e l, l, c j}$ and $V_{r e l, l, c j}$ (Equations (18) and (19))

Step 9: Identification of LOFA precursors

Figure 5. Flow chart of the OSSC procedure.

$$
\begin{aligned}
& \text { At } l \text {-th time } \\
& \text { Calculate } V_{1 s t}=\max _{c}\left(V_{\text {rel } l, l, c j}\right) \\
& \text { Calculate } c_{1 s t}=\arg \left(\max _{c}\left(V_{r e l, l, c j}\right)\right) \\
& \text { Calculate } V_{2 n d}=\max _{c \neq c_{1 s t}}\left(V_{r e l, l, c j}\right) \\
& \text { Calculate } c_{2 n d}=\arg \left(\max _{c \neq c_{1 s t}}\left(V_{\text {rel }, l, c j}\right)\right) \\
& \text { If }\left(V_{1 s t}>V_{\text {lim }, L O F A, l} \& V_{2 n d}>V_{\text {lim }, L O F A, l}\right) \\
& \text { Calculate } P_{L O F A, l, j} \text { with Equation }(20) \\
& \text { Else } \\
& P_{L O F A, l, j}=0 \\
& \text { End } \\
& \text { If } P_{L O F A, l, j}>P_{L O F A, l i m} \\
& \text { LOFA precursors are identified } \\
& \text { Else } \\
& \text { NO LOFA precursors are present } \\
& \text { End }
\end{aligned}
$$

Figure 6. Pseudo code for $P_{L O F A, l, j}$ analysis.

It is worth noting that $V_{\lim , \mathrm{LOFA}, l}$ and $P_{\mathrm{LOFA}, \mathrm{lim}}$ are determined from the training data, with the objective of minimizing the sum of false positives (i.e., not occurred failure events 
wrongly identified as occurred) and false negatives (i.e., occurred events not identified) encountered in the LOFA identification process on the training scenarios. In particular, the trend of $V_{\text {lim, LOFA,l }}$ is assumed to be a monotonic increasing piecewise function that is calculated relying on the pseudo-code of Figure 7 . The trend of $V_{\lim , L O F A, l}$ is initially defined using a discrete set of points obtained from the $N_{\text {data }}$ scenarios and then joined and corrected/revised to build a stepwise function which is monotonically increasing.

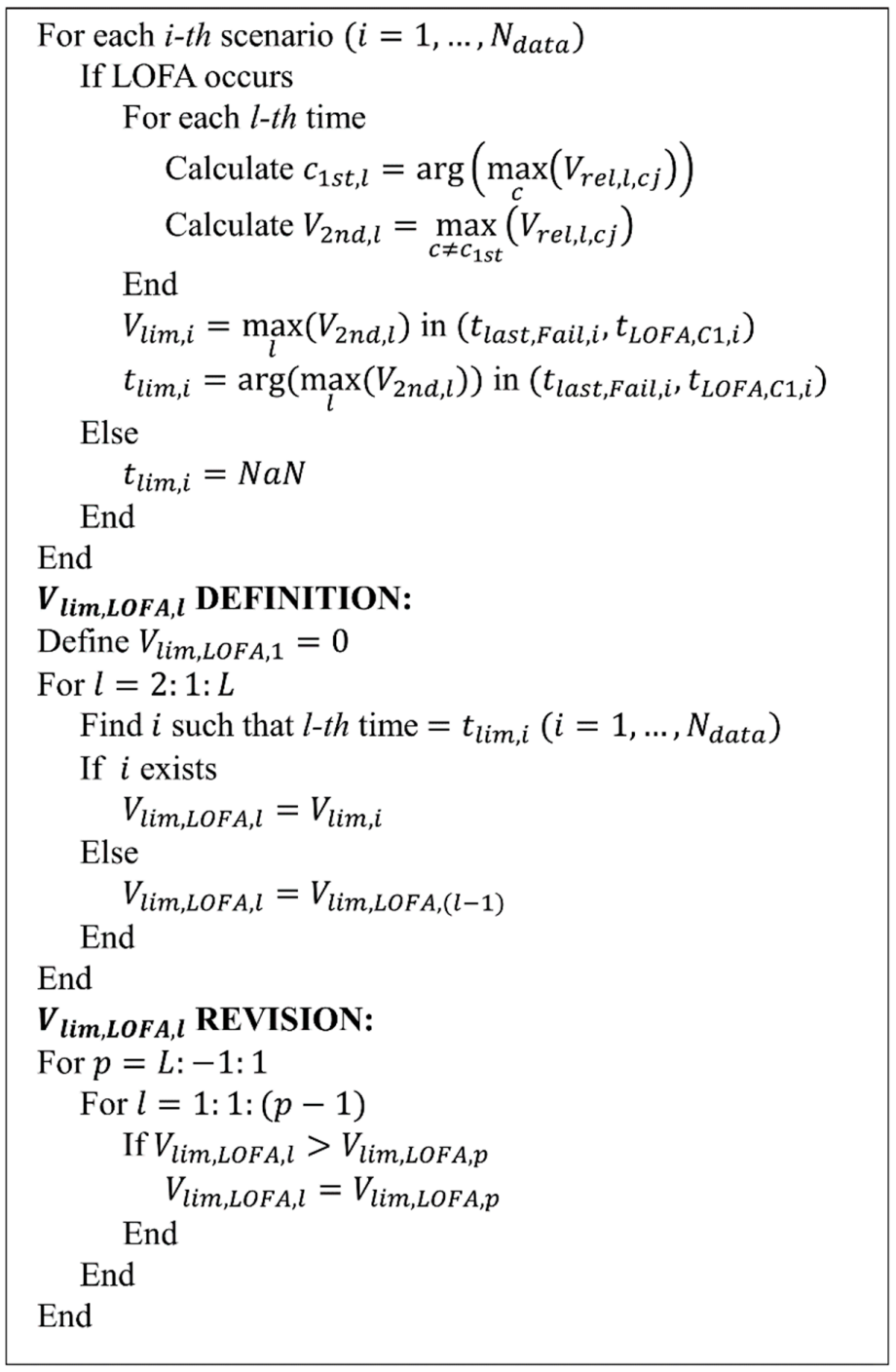

Figure 7. $V_{\text {lim }, L O F A, l}$ calculation procedure.

In Figure $7, t_{\text {last }, F a i l, i}$ is the time when the last failure before $t_{L O F A, C 1, i}$ occurs.

Step (9b): Compute $f_{C l, l, j}(c)$ and $P_{C l, l}(c)$ by Equation (21) and Equation (22), respectively, for each $c$-th cluster. The former is set equal to $P_{L O F A, l}(c)$ (see Equation (11)), only if the corresponding membership $M_{r e l, l, c j}$ overcomes the limit value $M_{l i m, F A I L, l}$ (see explanation and Equation (24) below); otherwise, it is set to 0 . The latter quantifies the degree with which cluster $c$ is "responsible" for the failure (i.e., the probability that a system state belonging to cluster $c$ is causing the LOFA). Actually, Equation (22) represents a "normalization" of Equation (11), conditional to the fact that a LOFA has occurred and has been identified. Then, $P_{F A I L, l, j}(e)$ is calculated for each $e$-th component, which represents the unconditional probability that in the $j$-th test scenario, component $e$ has failed at time $l$. The value of $P_{F A I L, l, j}(e)$ is obtained by the Theorem of Total probability, as the sum over all the clusters of the product between $P_{F A I L, l}(e \mid c)$ (estimated by Equation (12) and indicating the probability of failure of the $e$-th component at the $l$-th time conditional to the $c$-th cluster) 
and $P_{C l, l}(c)$ (see Equation (23) below). Finally, if $P_{F A I L, l, j}(e)$ exceeds $P_{F A I L, l i m}(e)$, then the $e$-th component is considered to have failed, as shown in the pseudo code of Figure 8.

$$
\begin{gathered}
f_{C l, l, j}(c)=\left\{\begin{array}{c}
P_{L O F A, l}(c) \text { if } M_{r e l, l, c j}>M_{l i m, F A I L . l} \\
0 \text { if } M_{r e l, l, l j} \leq M_{l i m, F A I L . l}
\end{array}\right. \\
P_{C l, l}(c)=\frac{f_{C l, l, j}(c)}{\sum_{c}^{C=9} f_{C l, l, j}(c)} \\
P_{F A I L, l, j}(e)=\sum_{c}^{C=9} P_{F A I L, l}(e \mid c) \times P_{C l, l, j}(c)
\end{gathered}
$$

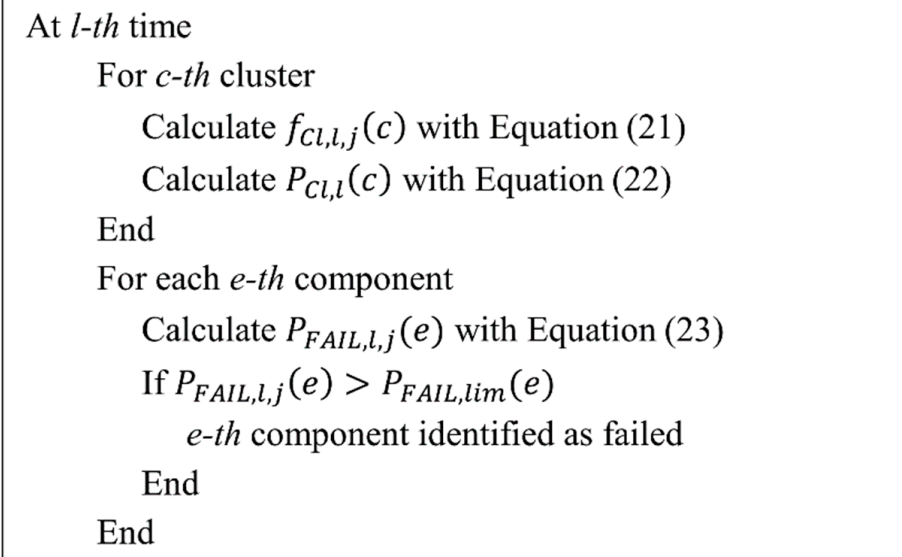

Figure 8. Pseudo code for $P_{F A I L, l, j}(e)$ analysis.

The trend of $M_{l i m, F A I L . l}$ and each $P_{F A I L, l i m}(e)$ are determined from the $N_{\text {data }}$ training scenarios, too. In particular, $M_{l i m, F A I L, l}$ is computed as in Equation (24):

$$
M_{\text {lim,FAIL.l }}=\mathcal{S} \times\left(l^{\text {th }} \text { time }\right)
$$

It is worth mentioning that $M_{l i m, F A I L, l}$ is assumed to be linearly dependent on time, because $\delta_{i j}$ (B.1) (Appendix B) linearly increases from $t=0 \mathrm{~s}$ to $t=t_{m i s s}=3600 \mathrm{~s}$ and it is used to calculate $M_{r e l, l, c j} . \mathcal{S}$ is the value that maximises the number of training scenarios whose components' failures are correctly identified as LOFA precursors, minimizing at the same time the time delay between component failures and precursor identification. Instead, for each $e$-th component, $P_{F A I L, l i m}(e)$ is set to minimize the sum between false positives and false negatives, related to the identification of the $e$-th component as precursor over the $N_{\text {data }}$ training scenarios.

\section{Results}

The main results of the overall procedure explained in Section 3 are here reported for the three steps. Finally, the devised method is tested on the $N_{\text {test }}=38$ scenarios different from the $N_{\text {data }}$ training ones to verify its robustness.

\subsection{Step 1: Creation of a "Database" of Simulated Accidental Scenarios}

The AK-MCS procedure (Figure 3) has been iterated three times employing the output $\left(I / I_{C R}\right)_{\max }(k=3)$ as reference: this has led to an increase of the scenarios in the database from $N_{\text {train }}=83$ (created with simple MCS and available from ref. [28]) to $N_{\text {krig }}=119$. Notice that $p_{C S M, i n, \max }(k=1)$ and $T_{H S, \max }(k=2)$ are not taken into account in AKMCS, because their values have been found to lie very far from the corresponding failure thresholds in the present case. 
For illustration purposes, the Kriging predictions $\hat{\boldsymbol{r}}_{M C S}^{3}\left(\overline{\boldsymbol{x}}_{M C S}\right)=\mathcal{M M}^{3}\left(\overline{\boldsymbol{x}}_{M C S}\right)=$ $\left\{\mathcal{M M}^{3}\left(\boldsymbol{x}_{1}\right), \ldots, \mathcal{M M}^{3}\left(\boldsymbol{x}_{N_{M C S}}\right)\right\}=\mu_{\hat{\gamma}^{3}}\left(\overline{\boldsymbol{x}}_{M C S}\right)=\left\{\mu_{\hat{\gamma}^{3}}\left(\boldsymbol{x}_{1}\right), \ldots, \mu_{\hat{\gamma}^{3}}\left(\boldsymbol{x}_{N_{M C S}}\right)\right\}$ of $\left(I / I_{C R}\right)_{\max }$ and their learning function values $\mathcal{U}^{3}\left(\boldsymbol{x}_{M C S}\right)$ for the $N_{M C S}=100,000$ configurations generated during different iterations of the AK-MCS algorithm are shown in Figure 9.
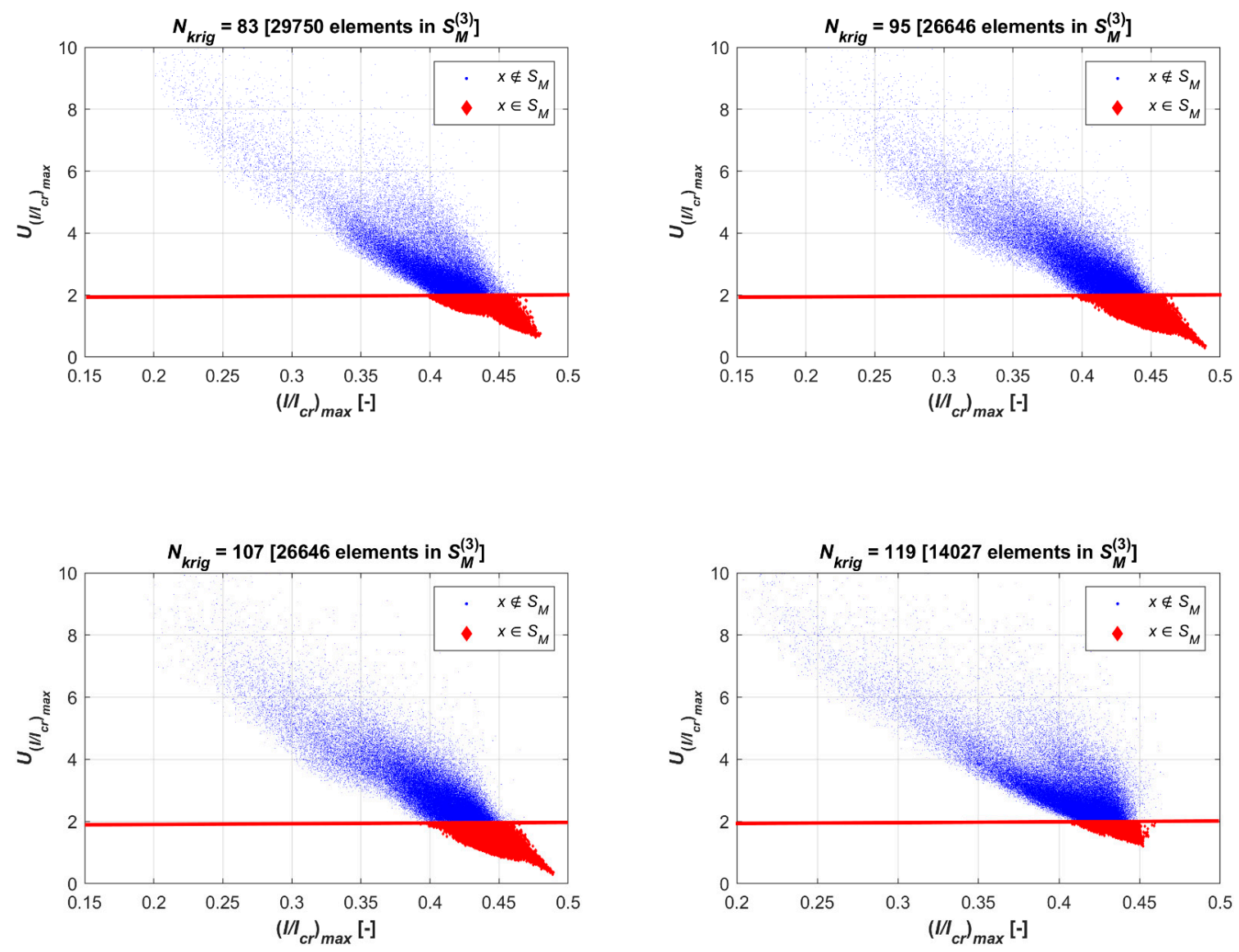

Figure 9. AK-MCS iterations for $\left(y_{i}^{3}(t)\right)_{\max }=\left(I / I_{c r}\right)_{\max }$.

A progressive decrease in the number of configurations contained in the $\alpha=95 \%$ confidence region $S_{\mathbb{M}}^{(3)}$ is evident at each step of the AK-MCS (red diamonds below the horizontal line, i.e., characterized by a value of the learning function $\mathcal{U}^{k}(\boldsymbol{X})$ (1) smaller than $\varepsilon=\Phi^{-1}\left(\frac{1+\alpha}{2}\right)=\Phi^{-1}(0.975)=1.96$ : see Section 3.1.1). They are halved after 3 iterations of the adaptive algorithm. In practice, this means that the area of the $S_{\mathbb{M}}^{(3)}$ region is reduced, i.e., that the precision (resp., uncertainty) of the Kriging metamodel in the characterization of the failure domain is increased (resp., decreased). Further, this demonstrates an improvement in the representativity of the abnormal (failure) scenarios in the dataset (in other words, a better coverage of the failure domain by the training dataset is obtained).

The $N_{\text {krig }}=119$ scenarios (simulated by the $4 \mathrm{C}$ code) are employed to train the POD-based Kriging metamodels. The accuracy of the constructed metamodels is estimated by: (i) a Leave-One-Out (LOO) assessment of the Normalized Root Mean Squared Error (NRMSE) computed on the training set of $N_{k r i g}=119$ transients; and (ii) an NRMSE evaluated on a test set of 38 scenarios never used during training (see Section 4.3): the corresponding error values are $4.73 \%$ and $7.94 \%$, respectively. The metamodel accuracy is satisfactory, considering that: (i) the size of the training set (containing only 119 transients) is quite small if referred to the dimensionality of the input space to map $(M=12)$ and 
to the functional nature of the outputs to be estimated (i.e., time-varying parameters); and (ii) the POD-based Kriging metamodels are not used here for carrying out a detailed thermal-hydraulic analysis for design purposes, but rather for quickly reproducing the main features of the SMCCC behavior and creating a large database of scenarios, on which the LOFA detection algorithms can rely. The metamodels thereby constructed have allowed simulating $N_{P O D}=700$ new time-varying transients with a sharp reduction in the computational burden with respect to the $4 \mathrm{C}$ code from an average of two days to about $1.1 \mathrm{~s}$ per simulation. The resulting (new) database is constituted by $N_{\text {data }}=819$ scenarios.

Figure 10 shows the POD-based Kriging prediction of the time evolution of the critical output variables for a scenario different from the $N_{\text {data }}$ ones. The POD-based Kriging metamodels can capture with satisfactory accuracy the time-varying behavior of the three critical variables. However, some considerations are in order with respect to the metamodel-based estimation of $I / I_{c r}$ (dashed line in Figure 10, bottom). The value of such parameters becomes smaller than zero between $410 \mathrm{~s}$ and $534 \mathrm{~s}$, which is not physical (in fact, $I / I_{c r} \geq 0$ always); also, in the last $200 \mathrm{~s}$ of the transient $I / I_{c r}$ seems to start increasing (instead of remaining equal to zero as in the reference solid curve produced by the $4 \mathrm{C}$ code). Such deviations from the real, physical behavior can be explained only by the expected regression errors introduced by metamodels trained with a very small-sized training set. In this view, such discrepancies could be reduced by increasing the number of training scenarios simulated by the $4 \mathrm{C}$ code (and correspondingly the computational cost of the analysis). Additionally, it is important to acknowledge that the relatively small entity of such non-physical deviations, combined with their very short duration with respect to the mission time ( $\tau_{\text {miss }}=3600 \mathrm{~s}$ ), still allows to quite accurately capture the global trend of the critical parameter over the time horizon of interest, on which the metamodel has been trained.
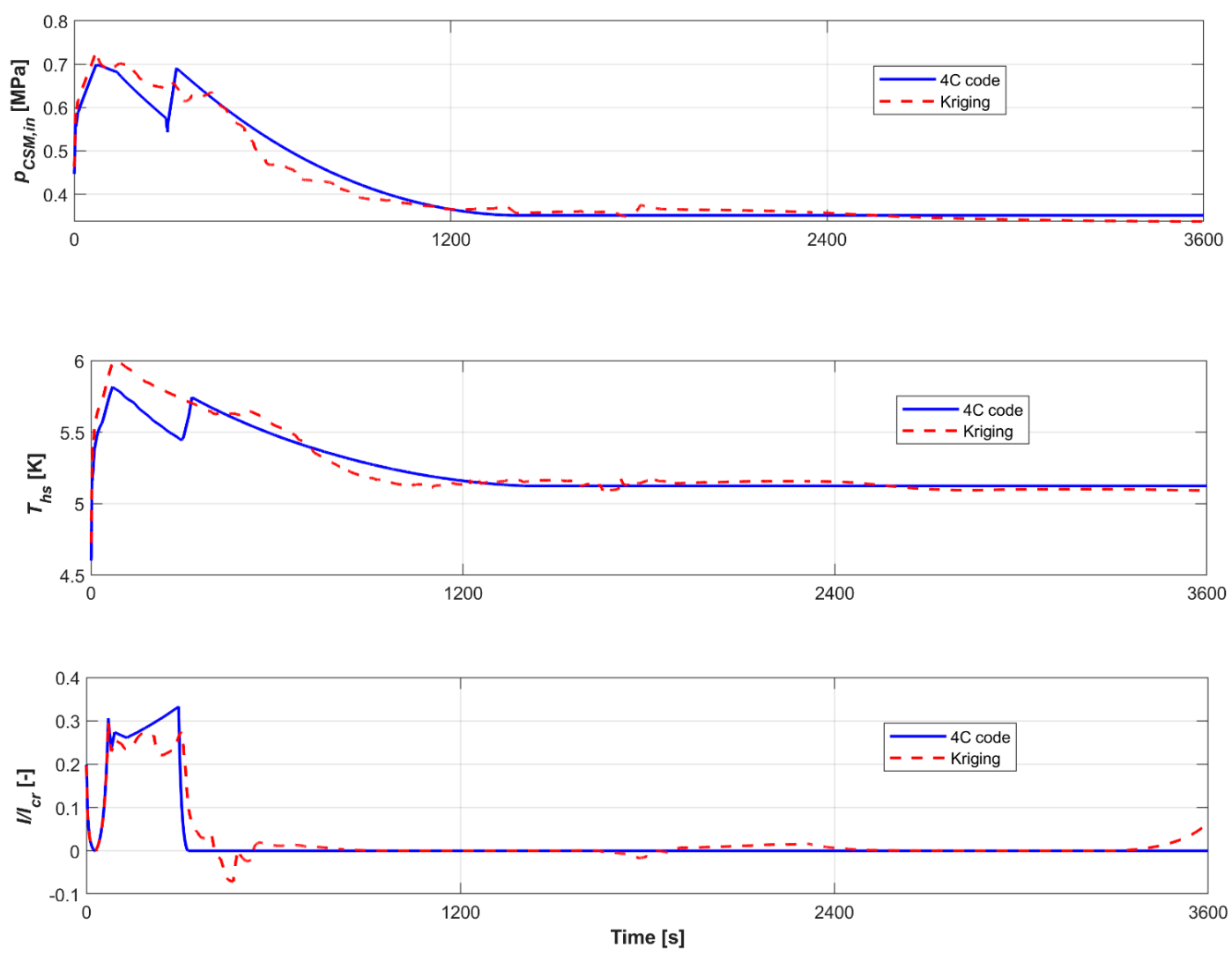

Figure 10. Comparison between the outputs obtained from the real $4 \mathrm{C}$ code (solid, blue) and the POD-based Kriging "surrogate" (dashed, red) for a scenario characterized by the partial opening of SV at $138 \mathrm{~s}$ and the complete closure of CV2 at $292 \mathrm{~s}$. 


\subsection{Step 2: Prototypical Transients and Components Failure Modes Identification}

Employing the unsupervised procedure of Section 3.2 and Appendix B, all the $N_{\text {data }}=$ $N_{\text {krig }}+N_{P O D}=819$ (4C- and metamodel-based) scenarios are optimally grouped into $C=9$ clusters. The behaviors of the critical variables $p_{C S M, i n}, T_{h s}$ and $I / I_{c r}$ for the $N_{\text {data }}$ scenarios, grouped in clusters, are sketched as dotted lines in Figures A2-A4, respectively, of Appendix D. For each cluster, the "prototypical" transient (i.e., the transient with the largest value of membership to the cluster) is also plotted (solid line) [79]. Cluster 4 is the most critical cluster showing the highest value of $p_{C S M, i n}$ and $T_{h s}$, which reach $0.8 \mathrm{MPa}$ and $6.3 \mathrm{~K}$, respectively. Notice that, however, the corresponding threshold limits $p_{\text {lim }}$ (i.e., $1.8 \mathrm{MPa}$ ) and $T_{c s}$ (i.e., $7.3 \mathrm{~K}$ ), respectively, are not overcome (in other words, $T_{h s}$ does not exceed the current sharing temperature $T_{c S}$ and the magnet does not lose its SC properties). From the system safety viewpoint, these simulation results are encouraging, since they show that in the present case (even in those configurations leading to a LOFA), none of the scenarios are really critical for the CS module's integrity.

Each cluster is characterized by different values of $P_{L O F A, l}(c)$ and of $P_{F A I L, l}(e \mid c)$, determined with Equation (11) and Equation (12) and sketched in Figures 11 and 12, respectively.

According to Figure 11, showing the probability of LOFA occurrence in time windows of width equal to $300 \mathrm{~s}$, a LOFA does not typically happen in configurations belonging to Clusters 2 and 7; it is more frequent in the interval [0 s, $900 \mathrm{~s}]$ for Cluster 4, [600 s, $900 \mathrm{~s}$ ] for Cluster 5, [600 s, $1500 \mathrm{~s}$ ] for Cluster 1, [1200 s, $1500 \mathrm{~s}$ ] for Cluster 8 and [1500 s, $2100 \mathrm{~s}$ ] for Cluster 6. The LOFA event is instead distributed along the entire time horizon for Clusters 3 and 9, with two peaks within [600 s, 900 s] and [1500 s, 1800 s], respectively, for the former and a peak in [1200 s, $1500 \mathrm{~s}]$ for the latter.
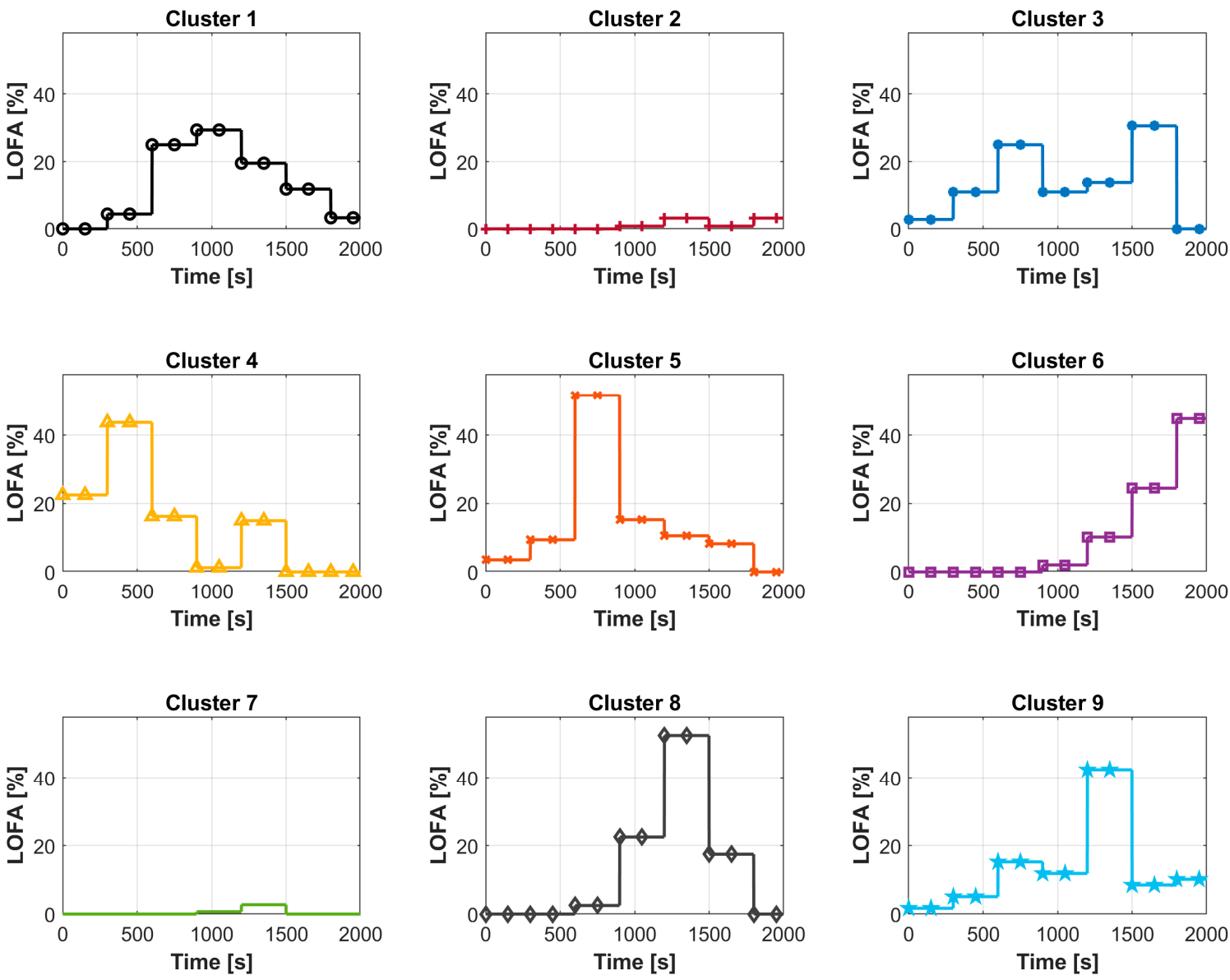

Figure 11. Probability of LOFA occurrence time $P_{L O F A, l}(c)$ (Equation (11)) for each cluster $c=1,2, \ldots, 9$. 
The conditional failure probability $P_{F A I L, l}(e \mid c)$ of each component $e$ for Cluster 4 as a function of time $l$ is reported in Figure 12, by way of example and only for illustration purposes. It can be inferred that if a LOFA occurs in a system configuration of Cluster 4 , it will likely be due to an "early" failure (i.e., within [0,600 s]) of either CP or CV1 or CV2 or BV (or combinations of such components). These results are coherent with the physics of the system and with the peculiar features of Cluster 4 (characterized by the occurrence of a LOFA and by the highest values of the current sharing temperature $T_{h s}$ ). Based a deeper, more detailed analysis (not reported here for brevity) of the $N_{\text {data }}=819$ simulated scenarios, the following considerations can be made. Similar to ref. [28], the BV being stuck open (in particular, at $50 \%$ of the nominal mass flow rate) is one of the most frequent failures in Cluster 4 (probabilities of $16 \%-21 \%$ in time intervals [ $0 \mathrm{~s}, 300 \mathrm{~s}$ ] and [300 s, $600 \mathrm{~s}$ ], respectively, as shown in Figure 12). As consequence of this failure, a smaller flow rate of $\mathrm{SHe}$ is available to remove heat from the CSM, which produces an increase in $T_{h s}$. However, it is worth remembering that a LOFA cannot occur as the result of the only failure of BV (stuck open at 50\%). Actually, as revealed by a closer inspection of all the 819 scenarios produced, after the BV failure the LOFA usually verifies in Cluster 4 because one component between the two CVs and the $\mathrm{CP}$ fails with the largest magnitude (i.e., $\mathrm{CV} 1$ or $\mathrm{CV} 2$ fails, being stuck completely closed, or CP decreases its rotational speed until it stops). Again, similar to ref. [28], other "prototypical" (and very frequent) failures in Cluster 4 cause a decrease in the helium mass flow rate available to remove heat from the CSM, determining the $T_{h s}$ increase. Such failures typically occur, again, at an early time in the accident scenario (i.e., within [0, $600 \mathrm{~s}]$ ), yet with a smaller magnitude. In particular, the CVs fail being stuck closed (at $50 \%$ of the nominal rate) (failure probability of $20 \%-23 \%$ in time interval [0 s, 600 s]; see Figure 12), whereas the CP reduces its rotational speed (from the nominal value to $75 \%-25 \%$ ) (failure probability of $13-34 \%$ in time interval [0 s, $600 \mathrm{~s}$ ]; see Figure 12). The conditional failure probabilities for the other clusters are not shown here for brevity.

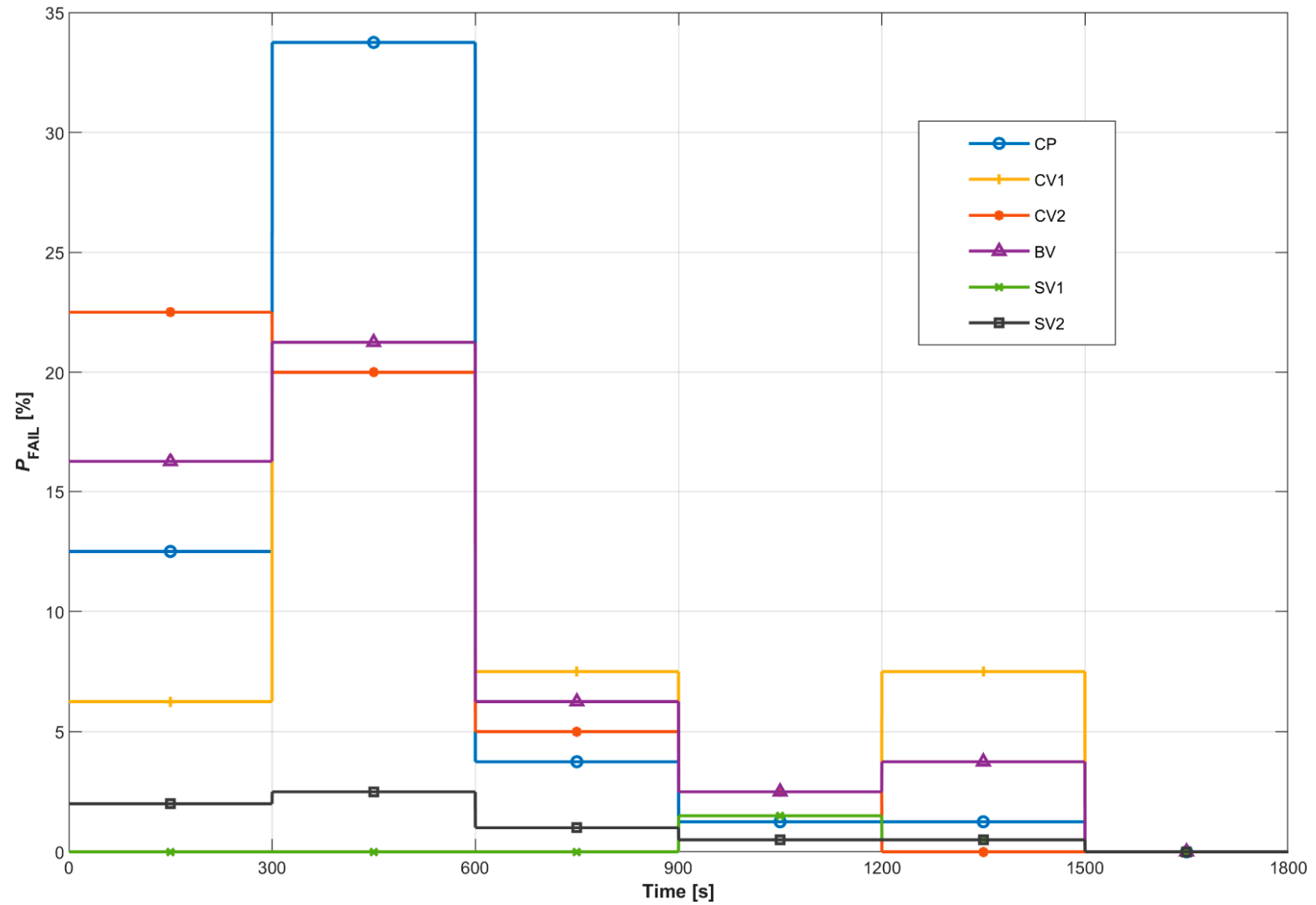

Figure 12. Conditional probability of failure $P_{F A I L, l}(e \mid c)$ of each component e for cluster $c=4$, as a function of time $l$. 
In conclusion, the most relevant output of the algorithmic step reported above is the identification of groups (i.e., clusters) and "prototypical configurations" of anomalous system evolutions (Figures 11 and 12). The importance resides in the fact that these prototypes can represent a basis for: (i) a prompt classification of new (evolving) transients as 'normal' or 'abnormal' (see Section 4.3 below); (ii) the pinpointing of components that are more likely to bring the system to critical states, and (iii) the subsequent scheduling and prioritization of inspection/maintenance interventions on these critical components (as highlighted, e.g., for CP, CV1, CV2 and BV with reference to Cluster 4). However, it must be remembered that for inspection/maintenance prioritization purposes, the operator should also take into account the likelihood of accident scenarios. In this work, scenarios have by construction equal likelihood. Instead, in real situations, their probabilities/frequencies can be very different; this obviously affects the components' importance/criticality ranking and, thus, the result of the related inspection/maintenance scheduling process.

\subsection{Step 3: Timely LOFA Precursors Identification by OSSC}

Firstly, the threshold values for the LOFA precursor identification algorithm, e.g., $V_{\text {lim,LOFA,l}}, P_{\text {LOFA,lim }}, M_{\text {lim }, F A I L . l}$ and $P_{F A I L, l i m}(e)[l=1,2, \ldots, L ; e=\mathrm{CP}, \mathrm{CV} 1, \mathrm{CV} 2, \mathrm{BV}, \mathrm{SV} 1$, SV2] must be properly set using the $N_{\text {data }}=819$ training scenarios. $V_{\text {lim }, L O F A, l}$ is obtained with the $N_{\text {data }}$ scenarios following the pseudo-code of Figure 7 and its time evolution is represented in Figure 13.

The coefficient $\mathcal{S}$ used to calculate $M_{l i m, F A I L, l}$ as in Equation (24) is set to $\mathcal{S}=9.81 \times 10^{-8} \mathrm{~S}^{-1}$, according to the "tuning" criterion reported at the end of Section 3 . The values of $P_{L O F A, l i m}$ and of each $P_{F A I L, l i m}(e)$ that are found to minimize the sum of false positive and false negatives in the LOFA precursor identification on the $N_{\text {data }}$ training scenarios are listed in Table 1.

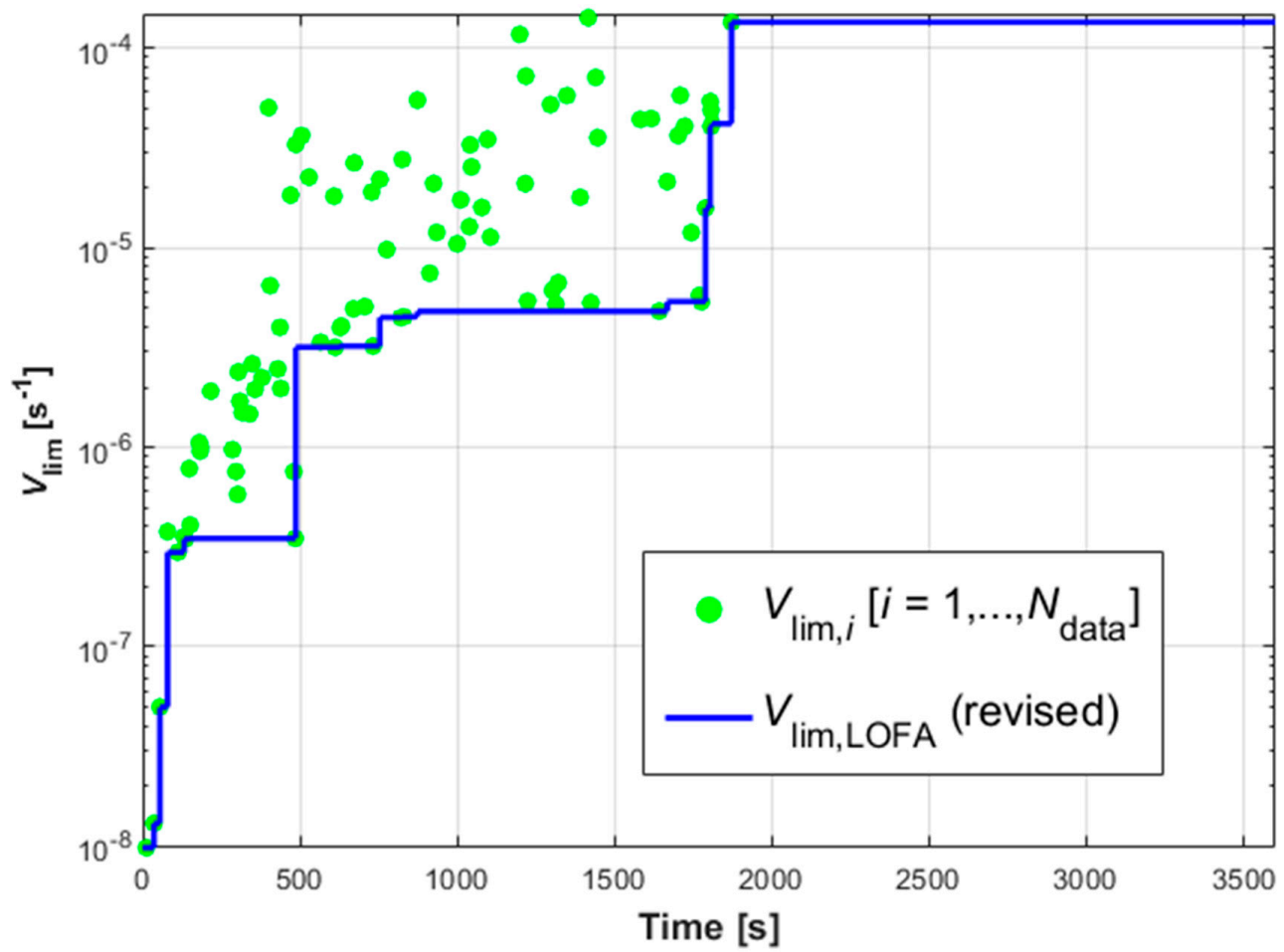

Figure 13. $V_{\text {lim }, L O F A, l}$ trend in logarithmic scale (blue line) and $V_{\text {lim }, i}$ values used to estimate it (green points). 
Table 1. Optimal values of $P_{L O F A, l i m}$ and $P_{F A I L, l i m}(e)$.

\begin{tabular}{cc}
\hline & Limit Value \\
\hline$P_{\text {LOFA,lim }}$ & $19 \%$ \\
$P_{\text {FAIL,lim }}(\mathrm{CP})$ & $8 \%$ \\
$P_{F A I L, \text { lim }}(\mathrm{CV} 1)$ & $10 \%$ \\
$P_{\text {FAIL,lim }}(\mathrm{CV} 2)$ & $3 \%$ \\
$P_{\text {FAIL,lim }}(\mathrm{BV})$ & $5 \%$ \\
$P_{\text {FAIL, lim }}(\mathrm{SV} 1)$ & $25 \%$ \\
$P_{\text {FAIL,lim }}(\mathrm{SV} 2)$ & $8 \%$ \\
\hline
\end{tabular}

Then, the "tuned" LOFA precursor identification algorithm is tested on $N_{\text {test }}=38$ scenarios, different from the $N_{\text {data }}=819$ ones used for training.

As an example, we show the scenario "partial closure of BV at $0 \mathrm{~s}, \mathrm{CP}$ speed at $75 \%$ at $1 \mathrm{~s}$, complete closure of CV2 at $71 \mathrm{~s}$ and complete closure of CV1 at $72 \mathrm{~s}$ ". The corresponding time evolutions of $M_{r e l, l, c j}$ and $V_{r e l, l, c j}(c=1, \ldots, C)$ are drawn in Figure 14 (left and right, respectively). Differently from all the other clusters, the membership to Cluster $4 M_{\text {rel }, l, 4 j}$ reaches a value very close to one (around 0.95) quite early in the transient (at about $t=590 \mathrm{~s}$ : see Figure 14, left). Additionally, such an increase is very rapid, as testified by the large positive peak in the discrete estimator of the derivative of the membership $M_{r e l, l, 4 j}$ (i.e., $V_{\text {rel }, l, 4 j}$ ) at about $t=470 \mathrm{~s}$ (Figure 14, right). Thus, even a visual inspection of Figure 14 qualitatively suggests that the scenario under analysis most likely belongs to Cluster 4 .

The quantitative information contained in $M_{r e l, l, c j}$ and $V_{r e l, l, c j}$ is rigorously elaborated according to the algorithms shown in Figure 6 and in Figure 8 and the results are reported in Figure 15. In particular, the values of $V_{r e l, l, c j}$ are used to calculate $P_{L O F A, l, j}$ (continuous blue line in Figure 15, top), which is compared to $P_{L O F A, l i m}$ (dashed line). The values of $M_{r e l, l, c j}$ are instead employed to determine $P_{F A I L, l, j}(e)$ (thick continuous lines in Figure 15, six bottom plots), which is compared to $P_{F A I L, l i m}(e)$ (dashed lines).
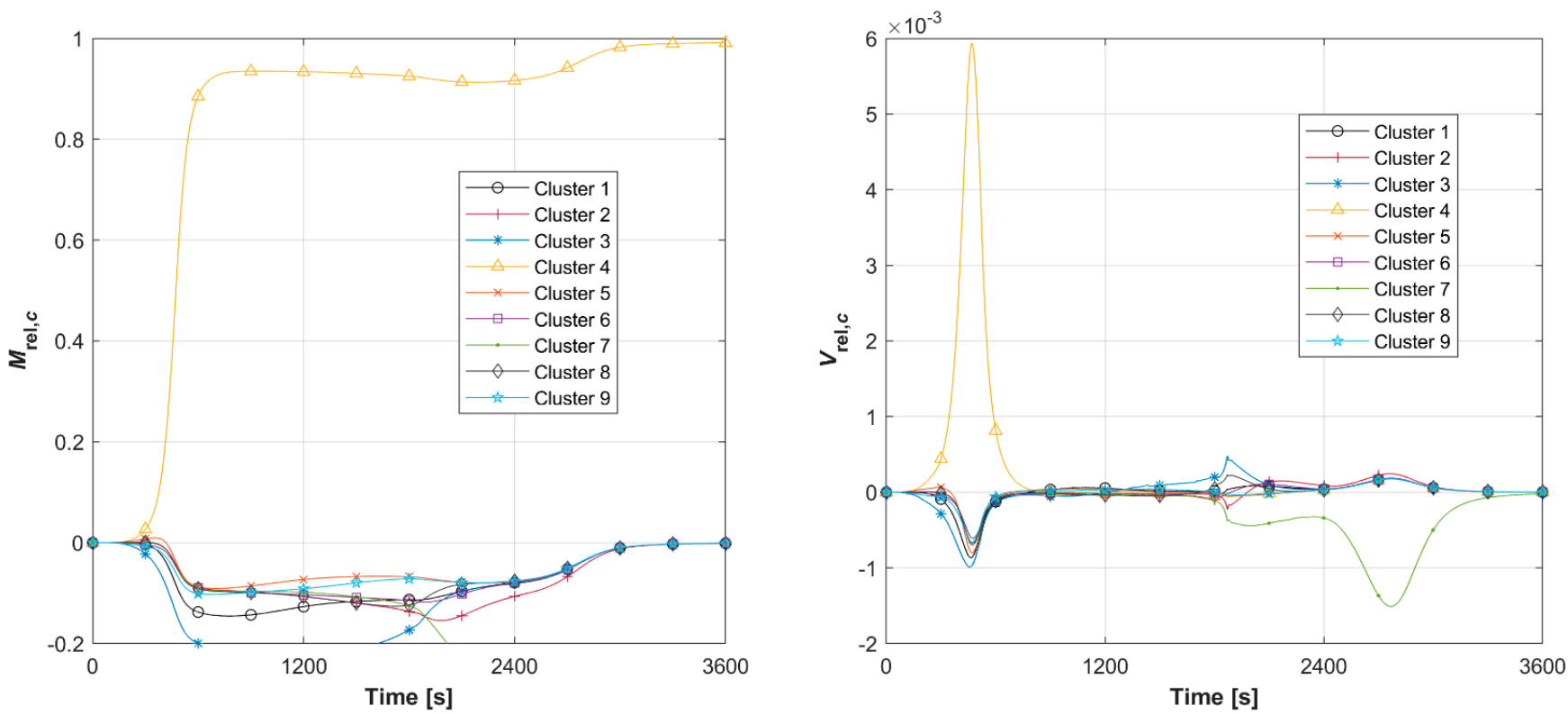

Figure 14. $M_{\text {rel,l,cj }}$ (left) and $V_{\text {rel l, },, c j}$ (right) trends for the scenario "partial closure of BV at $0 \mathrm{~s}, \mathrm{CP}$ speed at $75 \%$ at $1 \mathrm{~s}$, complete closure of CV2 at $71 \mathrm{~s}$ and complete closure of CV1 at $72 \mathrm{~s}$ ". 

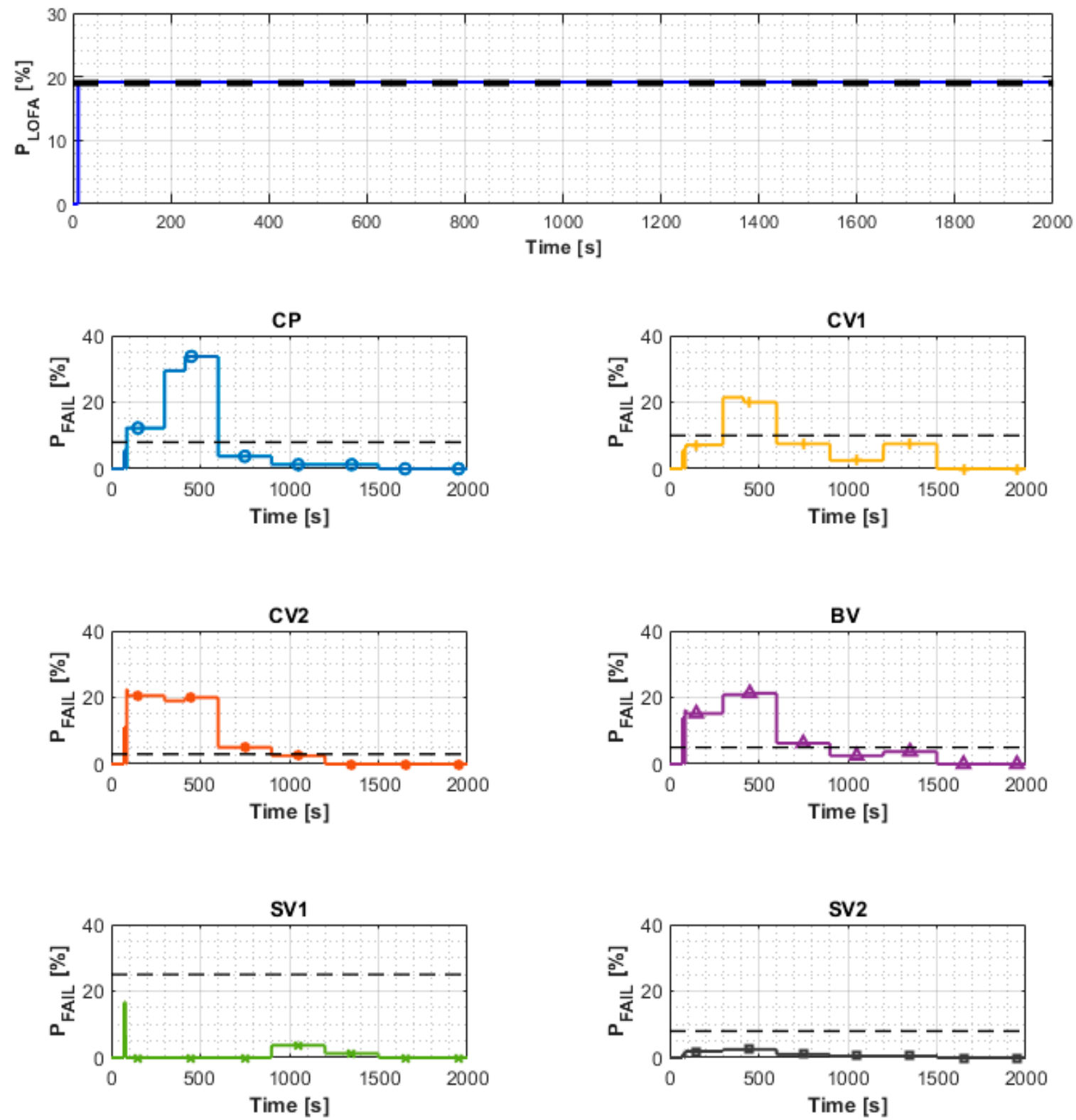

Figure 15. Values of $P_{L O F A, l, j}$ (top) and $P_{F A I L, l, j}(e)$ (six bottom plots) for scenario "partial closure of BV at $0 \mathrm{~s}, \mathrm{CP}$ speed at $75 \%$ at $1 \mathrm{~s}$, complete closure of CV2 at $71 \mathrm{~s}$ and complete closure of CV1 at $72 \mathrm{~s}$ " (continuous lines) compared with their limits $P_{\text {LOFA,lim }}$ and $P_{\text {FAIL,lim }}(e)$ (dashed lines).

At $11.2 \mathrm{~s}, P_{L O F A, l, j}$ overcomes $P_{L O F A, l i m}$ with a consequent LOFA identification; notice that the real time of LOFA occurrence (and detection) $t_{L O F A, C 1, j}$ for the scenario analyzed is $73.11 \mathrm{~s}$. At $72.6 \mathrm{~s}, P_{F A I L, l, j}(\mathrm{CV} 2)$ and $P_{F A I L, l, j}(\mathrm{BV})$ reach their corresponding limits, so that the CV2 and the BV are identified as precursors. At $87.2 \mathrm{~s}$, the $\mathrm{CP}$ is also identified as a precursor because $P_{F A I L, l, j}(\mathrm{CP})$ overcomes $P_{F A I L, l i m}(\mathrm{CP})$. Finally, $P_{F A I L, l, j}(\mathrm{CV} 1)$ reaches $P_{\text {FAIL, lim }}(\mathrm{CV} 1)$ at $300 \mathrm{~s}$ and its failure is also correctly identified. Instead, the SV1 and SV2 are considered "safe", because their failure probability indicator remains below the corresponding limit. In summary, the practical implications and benefits of using the proposed algorithm on this new, developing example scenario are the following: 
- $\quad$ The LOFA is detected $61.9 \mathrm{~s}$ earlier than the real occurrence time $t_{L O F A, C 1, j}=73.11 \mathrm{~s}$. This means that the online monitoring system developed is not only able to correctly detect and diagnose the abnormal state of the system (i.e., the presence of a LOFA), but also to predict it in advance: this allows the operators not only to implement the necessary and proper actions to address the peculiar transient condition, but also to have a sort of "grace period" to anticipate the system's accidental evolution and avoid more severe outcomes;

- $\quad$ CV2, BV, CP and CV1 are correctly identified as precursors (i.e., as root causes of the accident scenario) $1.6 \mathrm{~s}, 72.6 \mathrm{~s}, 86.2 \mathrm{~s}$ and $218 \mathrm{~s}$ later than their failures, respectively: this is important since it allows the operators to schedule specific and targeted maintenance interventions only for these faulty components;

- Finally, SV1 and SV2 are correctly identified as functioning: this avoids unnecessary inspections and interventions by the operators on safe components after the faulty condition of the machine has been detected.

In Table 2, the results of the extensive analysis performed with the LOFA precursor identification algorithm on the $N_{\text {test }}=38$ scenarios are summarized.

Table 2. LOFA identification results on $N_{\text {test }}=38$ scenarios.

$\begin{array}{cc}\text { Scenarios with LOFA } & 32 \\ \text { LOFA predicted in advance } & 24 \\ \text { LOFA not predicted in advance } & 8 \\ \text { Scenarios with NO LOFA } & 6 \\ \text { Correct identification NO LOFA } & 4 \\ \text { False positive LOFA } & 2\end{array}$

In 32 scenarios a LOFA occurs, whereas in six it does not. For the former ones, a LOFA is detected by the proposed algorithm before $t_{L O F A, C 1, j}$ in 24 scenarios and later in the other eight; however, in these last eight scenarios, the LOFA often occurs when no current flows into the CSM (i.e., in the time window [975 s, $1490 \mathrm{~s}]$ ), and the CSM is not endangered (obviously, the safety criterion based on the ratio $\left(I / I_{c r}\right)$ is very important when the current is flowing in the magnet, because it "reacts" very quickly in case of an anomaly. Instead, when there is no current flowing in the CSM $(I \approx 0 \mathrm{~A})$, the ratio $\left(I / I_{c r}\right)$ may seem to lose physical meaning and usefulness for LOFA detection purposes: this is technically true, but it does not represent a problem from the safety viewpoint, since the other two critical variables here monitored $\left(p_{C S M}\right.$,in and $\left.T_{h s}\right)$ are still able to warn the operator in case of abnormal behavior of the system). On the other hand, in four of the six scenarios with no LOFA, no LOFA precursors are correctly discovered, while in the other two a LOFA is detected even though it does not occur (namely, "false positives"). In general, a LOFA is predicted in advance in most scenarios with a negligible number of false positives in scenarios without a LOFA: globally, the elaborated method recognizes 95\% of the LOFA events and it can predict them in advance in $75 \%$ of the cases. This result can be practically interpreted as follows. By applying the proposed algorithmic framework to the cryogenic cooling circuit under analysis, in $95 \%$ of the cases we are able to correctly detect a LOFA, which allows us to take the necessary and proper actions to address this peculiar faulty condition. In addition, in $75 \%$ of the cases, we are also able to predict the LOFA in advance on the basis of the time behavior of the monitored critical variables, which provides us with a "time window" to anticipate the system accidental evolution and avoid more severe outcomes.

In Table 3, the results of precursor identification for the 32 scenarios with LOFA are reported. The first column (namely, "Correct precursor identification") contains the number of times a component actually fails in a given scenario and it is correctly identified as a precursor by the proposed algorithm. The second column (namely, "False negatives") reports the number of times a component actually fails in a given scenario, but it is incorrectly identified as safe. Conversely, the third column (namely, "Correct identification 
of normal operation") shows the number of times a component works without failures during its mission time, and it is correctly labelled as normally operating by our approach. Finally, the fourth column (namely, "False positives") contains the number of times a component operates normally in a given transient, but it is erroneously classified as failed.

Table 3. Results of the precursor identification approach for the 32 scenarios with LOFA.

\begin{tabular}{ccccc}
\hline Component & $\begin{array}{c}\text { Correct Precursor } \\
\text { Identification }\end{array}$ & False Negatives & $\begin{array}{c}\text { Correct Identification of } \\
\text { Normal Operation }\end{array}$ & False Positives \\
\hline CP & 21 & 2 & 2 & 7 \\
CV1 & 16 & 0 & 2 & 14 \\
CV2 & 13 & 2 & 9 & 8 \\
BV & 14 & 2 & 3 & 5 \\
SV1 & 1 & 1 & 25 & 8 \\
SV2 & 2 & 2 & 20 & \\
\hline
\end{tabular}

It can be seen that about $80 \%$ of the precursors are identified correctly by the OSSC algorithm, despite the large number of false positives for CP, CV1 and BV. This result can be practically interpreted as follows. By applying the proposed online monitoring technique to the SMCCC under analysis, we can correctly spot $80 \%$ of the failed components (i.e., the root causes of the LOFA scenario), which allows us to schedule specific and targeted maintenance interventions. In addition, it is worth mentioning that even if the percentage of false positives is still high (i.e., $56 \%$ ), this does not endanger the SMCCC, because conservatively overestimating the number of failed components (and, thus, the risk associated with the system). On the other side, it reduces its availability (due, e.g., to unnecessary inspections following the precursor identification).

Comparing the results above to those obtained by some of the authors in ref. [48], we observe a decrease from $67 \%$ to $33 \%$ in the erroneous LOFA identifications and a decrease from $80 \%$ to $56 \%$ in the erroneous precursor identification after a LOFA: in other words, the number of false positives has been reduced in a significant way (this is of paramount importance, since it dramatically decreases the number of unnecessary stops of the machine, each of which requires months to cool down the magnets). On the other hand, the percentage of times a LOFA is correctly identified in advance (with respect to its occurrence) has slightly diminished from $79 \%$ to $75 \%$, while the rate of correct precursor identifications has slightly decreased from $83 \%$ to $80 \%$, producing a slight increase in the "false negatives" (i.e., in the failed components that are not correctly identified). Overall, the percentage decrease in the "false positives" is much more significant than the increase in the "false negatives": in other words, the safety of the system analyzed is still satisfactorily guaranteed, but its unavailability (due, e.g., to unnecessary inspection procedures) is strongly reduced with respect to ref. [48]. A further increase in the accuracy of the metamodels and in the number of database scenarios used to feed the SC, FCM and OSSC algorithm will be certainly beneficial to an overall improvement of the proposed framework, both in terms of safety and availability.

\section{Conclusions}

In this work, a computational framework for the identification of abnormal conditions and of the corresponding precursors was proposed in three steps: (i) a "database" of simulated accident scenarios is created by Monte Carlo Sampling (MCS) and Proper Orthogonal Decomposition (POD)-based Kriging metamodels; (ii) the transients of the database are grouped in clusters according to their similarity through Spectral Clustering (SC) embedding the Fuzzy C-Means (FCM), in order to identify the principal patterns of system evolution towards failure and the "prototypical" precursors; (iii) the Online Supervised Spectral Clustering (OSSC) is employed to assign a new developing transient to one of the clusters previously discovered, thus enabling the identification of the precursors (i.e., failed components) of the abnormal conditions. 
For the first time, the devised method has been applied for the identification of the Loss-of-Flow Accident (LOFA) Precursors in a simplified Superconducting Magnet Cryogenic Cooling Circuit (SMCCC) that keeps one ITER Central Solenoid Module (CSM) cooled. The new approach has been tested on $N_{\text {test }}=38$ accidental scenarios to verify its robustness. Results have shown that $95 \%$ of the scenarios are correctly classified as "safe" or "faulty" and $80 \%$ of LOFA precursors are correctly identified. On the other hand, $56 \%$ of components that are not actually failed are identified as precursors (i.e., "false positives"). In this respect, three considerations must be made. First, from the system point of view, an important practical outcome of the analysis is the identification of groups (clusters) and "prototypical configurations" of anomalous behavioral patterns of the system, which is a basis for: (i) a prompt, online classification of new transients as 'normal' or 'abnormal' (in some cases, even for a prediction in advance of the anomalous system evolution); (ii) the identification of those components that are more relevant in determining the overall "health" state of the system and whose failure may drive the system into critical states with high likelihood; and (iii) the scheduling and prioritization of inspection/maintenance interventions on these important components. For example, for those scenarios belonging to Cluster 4, it is advisable to start the inspection/maintenance actions from CP, CV1, CV2 and BV. Second, thanks to the larger database of prototypical accident scenarios created by (fast-running) POD-based Kriging metamodels, the number of "false positives" has been significantly reduced with respect to a previous version of the algorithm proposed by some of the authors [48] (from $67 \%$ to $33 \%$ in the erroneous LOFA identifications and from $80 \%$ to $56 \%$ in the erroneous precursor identification conditional to LOFA scenarios). Third, a relatively high number of false positives does not endanger the SMCCC, because it conservatively overestimates the number of failed components (and, thus, the risk associated with the system). Due to the satisfactory results obtained, the proposed method may guide prioritization actions for inspection and maintenance of the SMCCC components. Additionally, notice that thanks to the use of metamodels, these satisfactory results have been obtained at the expense of very few (i.e., around 120) runs of the detailed, long-running simulation code.

Finally, in the future the following lines of research should be explored. The LOFA precursor identification algorithm should be refined by an increase in the accuracy of the POD-based metamodel together with an additional extension of the training database with more simulations, to: (i) further increase, if possible, the rate of correct LOFA detections, i.e., the percentage of scenarios correctly classified as "safe" or "faulty"; and (ii) most important, further reduce the number of "false positives" (actually, even if they do not impair the safety of the machine, they may cause an unacceptable unavailability level, due to the very long times - several months-typically needed to cool down the magnets at each stop). Additionally, the proposed approach should be adapted to larger-sized systems with more components, more severe operating conditions, and diversified accident scenarios (besides the LOFA). In this view, the sensitivity of its performance and generalization properties should be systematically tested also with respect to the numerous free parameters, on which our algorithms (POD-based metamodels, SC, FCM and OSSC) rely. The deployment of our algorithmic framework may boost the reliability, availability and maintainability of the plant, representing the basis for undertaking the proper prevention and mitigation strategies in the analysis of plants with a large volume of monitored signals.

Author Contributions: Conceptualization, V.D., N.P., R.B., F.D.M., L.S. and E.Z.; Formal analysis, V.D., N.P., R.B. and F.D.M.; Investigation, V.D., N.P., R.B. and F.D.M.; Methodology, V.D., N.P., R.B., F.D.M., L.S. and E.Z.; Software, V.D., N.P., R.B., F.D.M. and L.S.; Supervision, N.P., R.B., F.D.M. and E.Z.; Visualization, V.D., N.P. and F.D.M.; Writing-original draft, V.D. and N.P.; Writing-review and editing, V.D., N.P., R.B., F.D.M., L.S. and E.Z. All authors have read and agreed to the published version of the manuscript. 
Funding: This research received no external funding.

Institutional Review Board Statement: Not applicable.

Informed Consent Statement: Not applicable.

Data Availability Statement: Not applicable.

Conflicts of Interest: The authors declare no conflict of interest.

\section{Appendix A. Kriging Metamodels}

Kriging metamodels are stochastic regression algorithms, which allow predicting an output $\hat{Y}^{k}(\boldsymbol{X})=\mathcal{M M}^{k}(\boldsymbol{X})$ from an input $\boldsymbol{X} \in \mathcal{D}_{\mathcal{X}} \subset \mathbb{R}^{M}$ (with $M$ the number of input elements in $\mathcal{X}$, and $\mathcal{D}_{\mathcal{X}}$, the domain of $\boldsymbol{X}$ ) [39,73]. In Kriging metamodels, the residuals are correlated by mean of gaussian process like shown in Equation (A1):

$$
\mathcal{M M}^{k}(\boldsymbol{X})=\boldsymbol{\beta}_{k}{ }^{T} \boldsymbol{h}(\boldsymbol{X})+\sigma_{k}^{2} \mathcal{Z}\left(\boldsymbol{X}, \omega_{k}\right)=\mathcal{N}\left(\boldsymbol{\beta}_{k}{ }^{T} \boldsymbol{h}(\boldsymbol{x}) ; \sigma_{k}^{2} \mathcal{Z}\left(\boldsymbol{X}, \omega_{k}\right)\right)
$$

The first term of $\mathcal{M M}^{k}$ is the mean value (i.e., trend) of the linear regression model and it contains the regression coefficients $\beta_{k}=\left[\beta_{k 1}, \beta_{k 2}, \ldots, \beta_{k P}\right]$ and the basis function $\boldsymbol{h}=\left[\boldsymbol{h}_{1}, \boldsymbol{h}_{2}, \ldots, \boldsymbol{h}_{P}\right]$ where $P$ is the degree of the truncation of $\boldsymbol{h}(\boldsymbol{X})$. The second term is constituted by the variance of the gaussian process $\sigma_{k}^{2}$, a constant value, and by the zero mean unit variance $\mathcal{Z}\left(\boldsymbol{X}, \omega_{k}\right)$ of the gaussian process that depends on the input $\boldsymbol{X}$ and on the probability space $\omega_{k}$. The value $\omega_{k}$ relies on the correlation function $\mathcal{R}\left(\boldsymbol{X}, \boldsymbol{x}^{\prime} ; \boldsymbol{\theta}^{k}\right)$, depending on the distance between two input samples $\boldsymbol{X}$ and $\boldsymbol{X}^{\prime}$ and the hyperparameters $\theta^{k}$.

Each $\mathcal{M M}^{k}\left(k=1, \ldots, N_{k}\right)$ meta-model is built with the DOE made by the ensemble of the inputs $\overline{\boldsymbol{X}}=\left\{\boldsymbol{x}_{1}, \boldsymbol{x}_{2}, \ldots, \boldsymbol{x}_{N_{k r i g}}\right\}$ and the ensemble of the outputs $\boldsymbol{y}^{k}=$ $\left\{\mathcal{Y}_{1}^{k}, \mathcal{Y}_{2}^{k}, \ldots, \mathcal{Y}_{N_{k r i g}}^{k}\right\}$ both constituted by $N_{k r i g}$ elements. Consequently, to the assumption of the gaussian process, the $k$-th element output prediction $\hat{Y}^{k}(\boldsymbol{X})=\mathcal{M M}^{k}(\boldsymbol{X})$ for a general $\boldsymbol{X}$ input and all the true model responses $\boldsymbol{y}^{k}$ of the DOE follows the gaussian distribution in Equation (A2):

$$
\left[\begin{array}{c}
\hat{Y}^{k}(\boldsymbol{x}) \\
\boldsymbol{y}^{k}
\end{array}\right] \sim \mathcal{N}_{N_{k r i g}+1}\left(\left[\begin{array}{c}
\boldsymbol{\beta}_{k}{ }^{T} \boldsymbol{h}(\boldsymbol{x}) \\
\boldsymbol{H} \boldsymbol{\beta}_{k}
\end{array}\right] ; \sigma_{k}^{2}\left[\begin{array}{cc}
1 & \boldsymbol{r}_{k}^{T}(\boldsymbol{x}) \\
\boldsymbol{r}_{k}(\boldsymbol{x}) & \boldsymbol{\mathcal { R }}^{k}
\end{array}\right]\right)
$$

where:

$\mathcal{H}$ is the information matrix, whose generic term is $\left(i=1, \ldots, N_{\text {krig }} ; p=1, \ldots, P\right)$

$$
\mathcal{H}_{i p}=h_{p}\left(\boldsymbol{x}_{i}\right)
$$

$\boldsymbol{\mathcal { R }}^{k}$ is the correlation matrix, whose generic term is $\left(i, j=1, \ldots, N_{\text {krig }}\right)$

$$
\mathcal{R}_{i j}^{k}=\mathcal{R}\left(\boldsymbol{x}_{i}, \boldsymbol{x}_{j} ; \boldsymbol{\theta}^{k}\right)
$$

$\boldsymbol{r}_{k}(\boldsymbol{X})$ is vector of cross-correlations between the configuration $\boldsymbol{X}$ and each one of the DOE and its generic term is $\left(i=1, \ldots, N_{\text {krig }}\right)$

$$
r_{k i}(\boldsymbol{X})=\mathcal{R}\left(\boldsymbol{X}, \boldsymbol{x}_{i} ; \boldsymbol{\theta}^{k}\right)
$$


Consequently, the mean Kriging value $\mu_{\hat{\Upsilon}^{k}}$ at the $\mathcal{X}$ point and its Kriging variance $\sigma_{\hat{\Upsilon}^{k}}^{2}$ are estimate with Equations (A6) and (A7), respectively:

$$
\begin{aligned}
& \mu_{\hat{\Upsilon}^{k}}(\boldsymbol{X})=\boldsymbol{h}(\boldsymbol{X}) \boldsymbol{\beta}_{k}+\boldsymbol{r}_{k}^{T}(\boldsymbol{X}) \boldsymbol{R}^{k}-1\left(\boldsymbol{y}^{k}-\mathcal{H}_{\beta_{k}}\right) \\
& \sigma_{\hat{Y}^{k}}^{2}(\boldsymbol{X})=\sigma_{k}^{2}\left(1-\boldsymbol{r}_{k}^{T}(\boldsymbol{X}) \boldsymbol{\mathcal { R }}^{k}-1 \boldsymbol{r}_{k}(\boldsymbol{X})+\boldsymbol{u}_{k}^{T}(\boldsymbol{X})\left(\mathcal{H}^{T} \mathcal{R}^{k}-1 \boldsymbol{\mathcal { H }}\right)^{-1} \boldsymbol{u}_{k}(\boldsymbol{X})\right) \\
& \text { with } \boldsymbol{u}_{k}(\boldsymbol{X})=\boldsymbol{H}^{T} \boldsymbol{R}^{k}-1 \boldsymbol{r}_{k}(\boldsymbol{X})-\boldsymbol{h}(\boldsymbol{X}) \\
& \text { and } \boldsymbol{\beta}_{k}=\left(\boldsymbol{H}^{T} \mathcal{R}^{k-1} \mathcal{H}\right)^{-1} \mathcal{H}^{T} \boldsymbol{R}^{k-1} \boldsymbol{y}^{k}
\end{aligned}
$$

In order to obtain the $\mathcal{M M}^{k}$ Kriging meta-model, some steps are necessary: (i) Choose the trend basis function $\boldsymbol{h}(\boldsymbol{x})$ to adopt for $\mathcal{M M}^{k}$; (ii) Choose an opportune correlation function $\mathcal{R}\left(\boldsymbol{x}_{i}, \boldsymbol{x}_{j} ; \boldsymbol{\theta}^{k}\right)$; (iii) Set the hyperparameters $\boldsymbol{\theta}^{k}$, necessary for the evaluation of the gaussian variance $\sigma_{\hat{\gamma}^{k}}^{2}$ and of the regression coefficients $\beta_{k}$, or choose a method to find the optimum $\boldsymbol{\theta}^{k}$.

In this work, ordinary Kriging metamodels are developed, which means that the trend basis $\boldsymbol{\beta}_{k}^{T} \boldsymbol{h}(\boldsymbol{X})$ is considered constant $\left[\boldsymbol{\beta}_{k}^{T} \boldsymbol{h}(\boldsymbol{X})=\beta_{k, 1} \boldsymbol{h}_{1}(\boldsymbol{X})=\beta_{k, 0}\right.$, with $\left.\boldsymbol{h}_{1}(\boldsymbol{X})=1\right]$. The correlation function $\mathcal{R}\left(\boldsymbol{X}, \boldsymbol{X}^{\prime} ; \boldsymbol{\theta}^{k}\right)$ used in the model is "ellipsoidal" and "anisotropic" like in Equation (A10):

$$
\begin{gathered}
\mathcal{R}\left(\boldsymbol{x}, \boldsymbol{x}^{\prime} ; \boldsymbol{\theta}^{k}\right)=\mathcal{R}\left(\xi^{k}\right) \\
\text { with } \xi\left(\boldsymbol{x}, \boldsymbol{x}^{\prime} ; \boldsymbol{\theta}^{k}\right)=\sqrt{\sum_{m=1}^{M}\left(\frac{\mathcal{X}_{m}-\mathcal{X}_{m}^{\prime}}{\theta_{m}^{k}}\right)^{2}}
\end{gathered}
$$

where $\xi$ is an ellipsoidal function. Precisely, a "3/2 Matérn" correlation function (A12) is used in the Gaussian Process:

$$
\mathcal{R}(\xi)=(1+\xi \sqrt{3}) e^{-\xi \sqrt{3}}
$$

A Kriging optimization method is needed to calculate the hyperparameters $\theta^{k}$ necessary for the definition of $\sigma_{k}^{2}$. For this purpose, the $K$-fold cross validation embedding the Hybrid Genetic Algorithm (HGA) is adopted to find the minimum value of the optimization function (A13):

$$
\boldsymbol{\theta}^{k}=\underset{\mathcal{D}_{\theta}}{\operatorname{argmin}} \sum_{i=1}^{N_{k r i g}}\left(\mathcal{M M}^{k}\left(\boldsymbol{x}_{i}\right)-\mu_{\hat{\Upsilon}^{k},(-i)}\left(\boldsymbol{x}_{i}\right)\right)^{2}
$$

where $\mu_{\hat{\Upsilon}^{k},(-i)}\left(\boldsymbol{X}_{i}\right)$ is the mean value at the point $\boldsymbol{X}_{i}$ of the Kriging predictor built with all the DOE excepting for the couple $\left(\boldsymbol{x}_{i}, \boldsymbol{y}_{i}\right)$.

Once the HGA find the optimum value of each $\theta_{m}^{k}(m=1,2, \ldots, M)$ in the range $[0.001,10]$, the variance $\sigma_{k}^{2}$ is defined as in Equation (A14):

$$
\sigma_{k}^{2}=\frac{1}{N_{k r i g}} \sum_{i=1}^{N_{k r i g}} \frac{\left(\mathcal{M M}^{k}\left(\boldsymbol{x}_{i}\right)-\mu_{\hat{\Upsilon}^{k},(-i)}\left(\boldsymbol{x}_{i}\right)\right)^{2}}{\sigma_{\hat{\Upsilon}^{k},(-i)}^{2}\left(\boldsymbol{x}_{i}\right)}
$$

where $\sigma_{\hat{Y}^{k},(-i)}^{2}\left(\boldsymbol{X}_{i}\right)$ is the variance at the point $\boldsymbol{X}_{i}$ of the Kriging predictor built with all the DOE excepting for the couple $\left(\boldsymbol{x}_{i}, \boldsymbol{y}_{i}\right)$. 
For each $k$-th output, the meta-model $\mathcal{M M}^{k}$ is trained $N_{\text {iter }}=100$ times and the one with the lowest Leave-One-Out (LOO) error will be the $\mathcal{M M}^{k}$ chosen to mimic the behaviour of the $k$-th output. The LOO error is evaluated with Equation (A15):

$$
L O O=\frac{1}{N_{k r i g}} \sum_{i=1}^{N_{k r i g}} \frac{\left(\mathcal{M M}^{k}\left(\boldsymbol{x}_{i}\right)-\mu_{\hat{Y},(-i)}\left(\boldsymbol{x}_{i}\right)\right)^{2}}{\sigma^{2}}
$$

\section{Appendix B. Spectral Clustering (SC) Embedding the Fuzzy C-Means (FCM)}

Spectral Clustering (SC) embedding the Fuzzy C-Means (FCM) let to cluster $N$ objects through the following steps [28] (the flowchart of the algorithm is represented in Figure A1 for the sake of clarity):

- $\quad$ Step (1): The matrix $\overline{\bar{Y}}^{k}[N, L]$ is built for each $k$-th variable considered, collecting at each row all the $N$ transients associated with that variable for the $L$ time length and its generic element is $y_{i l}^{k}(i=1,2, \ldots, N ; l=1,2, \ldots, L)$ referring to the $i$-th scenario at the $l$-th time.

- Step (2): The matrix $\stackrel{=}{Y}[N, L]$ is normalized in the range $[0.2,0.8]$ obtaining the matrix $\overline{\bar{Y}}_{n}^{k}[N, L]$, whose generic element is $y_{n, i l}^{k}$

- $\quad$ Step (3): The Euclidean pointwise distance $\delta_{i j}$ between an $i$-th object and a $j$-th one $(j=1,2, \ldots, N)$ is determined as in Equation (A16):

$$
\delta_{i j}=\sum_{k=1}^{Z} \sum_{l=1}^{L}\left|y_{n, i l}^{k}-y_{n, j l}^{k}\right|
$$

- $\quad$ Step (4): The generic element $w_{i j}$ of the similarity matrix $\overline{\bar{W}}[N, N]$ is obtained from $\delta_{i j}$ as follows in Equation (A17):

$$
w_{i j}=e^{-F \times \delta_{i j}^{2}}
$$

with $F=1.7 \times 10^{-9}[6]$.

The similarity $w_{i j}$ can assume values between 0 and 1 : if it is close to 1 , the $i$-th and the $j$-th objects considered are very similar; instead, if it is near 0 , the two objects are very different.

- $\quad$ Step (5): The matrix $\overline{\bar{L}}_{s y m}[N, N]$, that is the Normalized Laplacian matrix associated with $\overline{\bar{W}}$, is computed. Then, to obtain the optimal number $C$ of groups in which the $N$ transients must be clustered, the eigenvalues $\lambda_{1}, \lambda_{2}, \ldots, \lambda_{N}$ of $\overline{\bar{L}}_{s y m}[N, N]$ and their associated eigenvectors $\vec{u}_{1}, \vec{u}_{2}, \ldots, \vec{u}_{N}$ are extracted. Following the eigengap heuristic theory, $C$ is set equal to the number of eigenvalues $\lambda_{1}, \lambda_{2}, \ldots, \lambda_{C}$ that are significantly smaller than $\lambda_{C+1}$ [75]. Each eigenvector $\vec{u}_{c}(c=1, \ldots, C)$ constitutes the $c$-th column of the matrix $\overline{\bar{U}}[N, C]$, that is normalized calculating the matrix $\overline{\bar{T}}[N, C]$, whose generic element $t_{i c}(i=1,2, \ldots, N, c=1,2, \ldots, C)$ is determined as follow in Equation (A18):

$$
t_{i c}=\frac{u_{i c}}{\sqrt{\sum_{c=1}^{C} u_{i c}^{2}}}
$$

- Step (6): The matrix $\overline{\bar{T}}$, which contains the features extracted of the $N$ objects, is fed to the FCM code [28] to group them in $C$ clusters. 


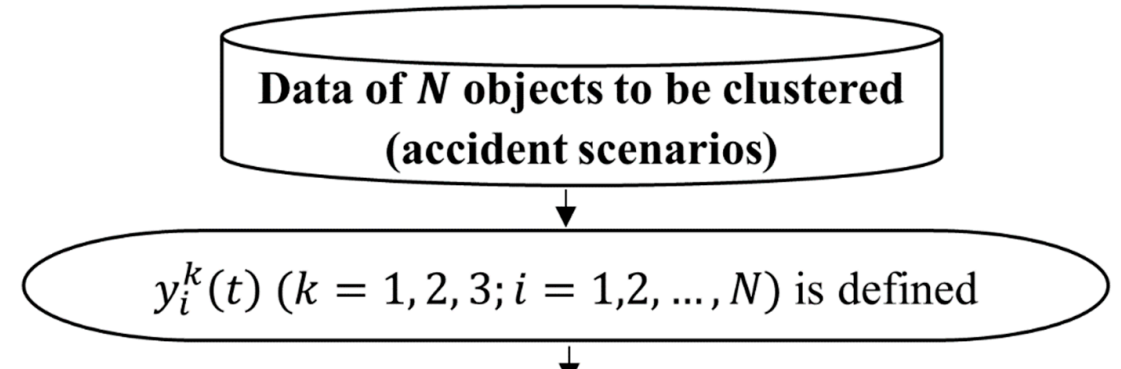

Step 1: Record $y_{i l}^{k}$ from $y_{i}^{k}(t)(l=1, \ldots, L)$ and build matrix $\overline{\bar{Y}}^{k}[N, L]$ for each $k$-th critical variable considered

$\downarrow$

Step 2: Normalize the elements of matrix $\overline{\bar{Y}}^{k}[N, L]$ in the range $[0.2,0.8]$ to obtain matrix $\overline{\bar{Y}}_{n}^{k}[N, L]$ with elements $y_{n, i l}^{k}$

Step 3: Calculate the Euclidean pointwise distance $\delta_{j i}$ between a $j$-th object (scenario) and an $i$-th object (scenario) $(i, j=1,2 \ldots, N)$ using (A.16)

Step 4: Build the similarity matrix $\overline{\bar{W}}[N, N]$, whose elements $w_{i j}$ are computed using (A.17)

Step 5: Calculate matrix $\overline{\bar{T}}[N, C]$ by normalizing $\overline{\bar{U}}[N, C]$, which is found by projecting $\overline{\bar{W}}[N, N]$ in the eigenspace coordinates with Equation (A.19)

Step 6: Use the FCM code to obtain two matrices:

- $\quad \overline{\overline{\mathcal{A}}}[C, C]$ containing the eigenspace coordinates of the centers of the $C$ clusters optimally identified

- $\quad \overline{\overline{\mathcal{M}}}\left[C, N_{\text {data }}\right]$ containing the membership degrees of each object (transient) to each cluster

\section{STOP}

Figure A1. Flowchart of the Spectral Clustering (SC) embedding the Fuzzy C-Means (FCM).

\section{Appendix C. Supervised Spectral Clustering: Projection in Eigenspace}

The similarity vector $\bar{W}_{l, j}$ must be projected in the eigenspace coordinates, contained in the vector $\bar{T}_{l, j}[1, C]$, to find the membership degree $M_{l, c j}$ respect to the $c$-th cluster at l-th time step: indeed, the value $M_{l, c j}$ can be estimated comparing $\bar{T}_{l, j}[1, C]$ and $\overline{\mathcal{A}}_{c}[1, C]$, containing the eigenspace coordinates of the prototypical cluster $c$ (Equation (17)).

Firstly, the vector $\bar{U}_{l, j}[1, C]$ is computed evaluating each element $u_{l, j c}(c=1,2, \ldots, C)$, as in Equation (A19): 


$$
\begin{gathered}
u_{l, j c}=\frac{d_{l, j}-1 / 2}{1-\lambda_{c}} \bar{W}_{l, j} \bar{D}^{-1 / 2} \vec{u}_{c} \\
\text { with } d_{l, j}=\sum_{i=1}^{N_{d a t a}} w_{l, j i}
\end{gathered}
$$

where $\lambda_{c}$ and $\vec{u}_{c}$ are the $c$-th eigenvalue and eigenvector of the matrix $\overline{\bar{L}}_{\text {sym }}$ of Section 3.2, respectively, and $\overline{\bar{D}}$ is the Degree matrix $\overline{\bar{D}}\left[N_{\text {data }}, N_{\text {data }}\right]$, which is a diagonal matrix composed by each element $d_{i}\left(i=1, \ldots, N_{\text {data }}\right)$ determined with Equation (A21) with the elements of the similarity matrix $\overline{\bar{W}}$ of Section 3.2:

$$
d_{i}=\sum_{j=i}^{N_{\text {data }}} w_{i j}
$$

Then, $\bar{U}_{l, j}$ is normalized, determining $\bar{T}_{l, j}[1, C]$, whose generic element $t_{l, j c}(c=1, \ldots, C)$ is given by Equation (A22):

$$
t_{l, j c}=\frac{u_{j, j c}}{\sqrt{\sum_{c=1}^{C} u_{j, j c}^{2}}}
$$

Appendix D. Nine Clusters of the Three Critical Variables Transient Scenarios, Generated by POD-Based Kriging Metamodels
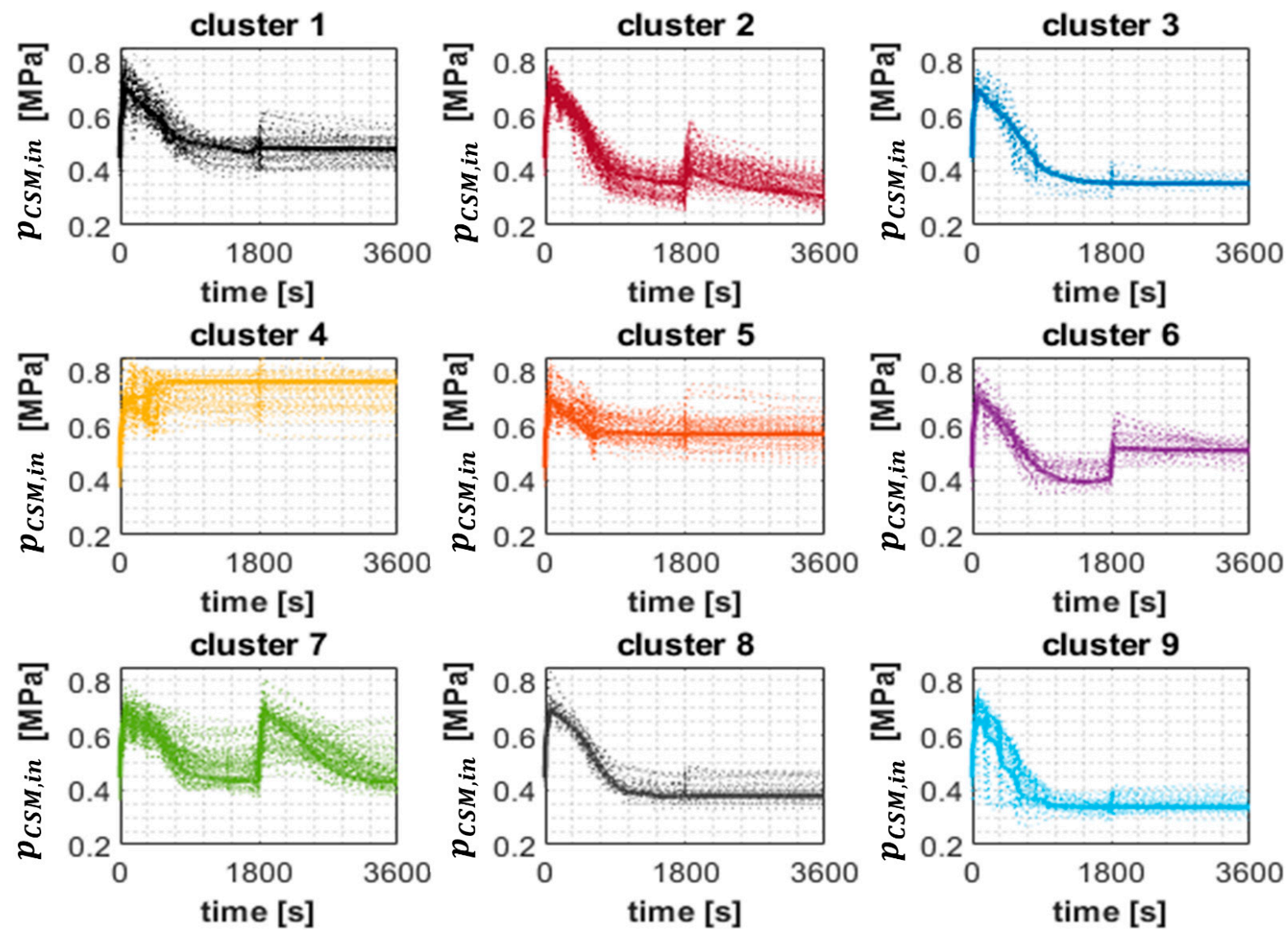

Figure A2. Nine clusters of $p_{C S M, \text { in }}$ transient scenarios generated by POD-based Kriging metamodels. 

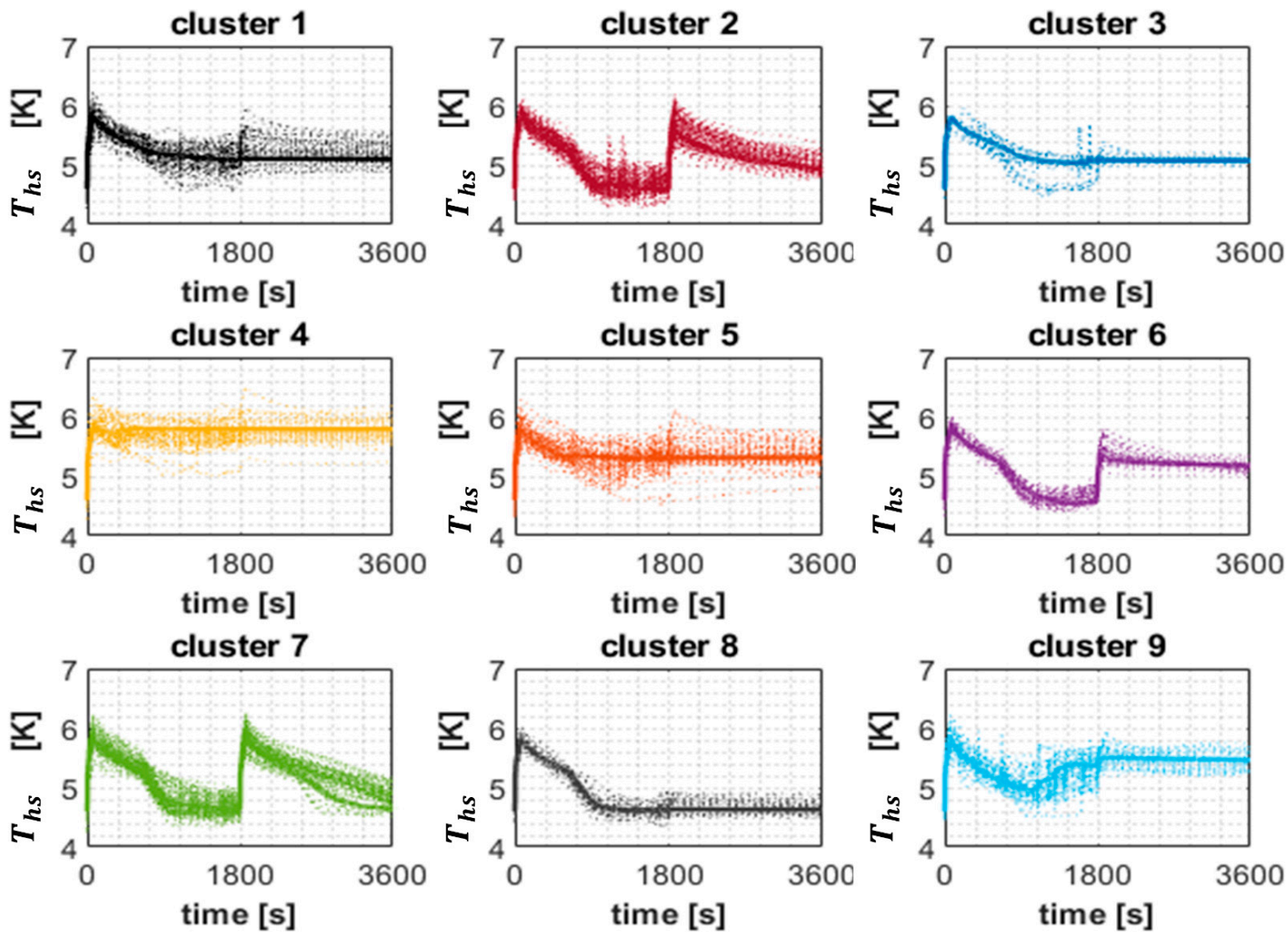

Figure A3. Nine clusters of $T_{h s}$ transient scenarios generated by POD-based Kriging metamodels.
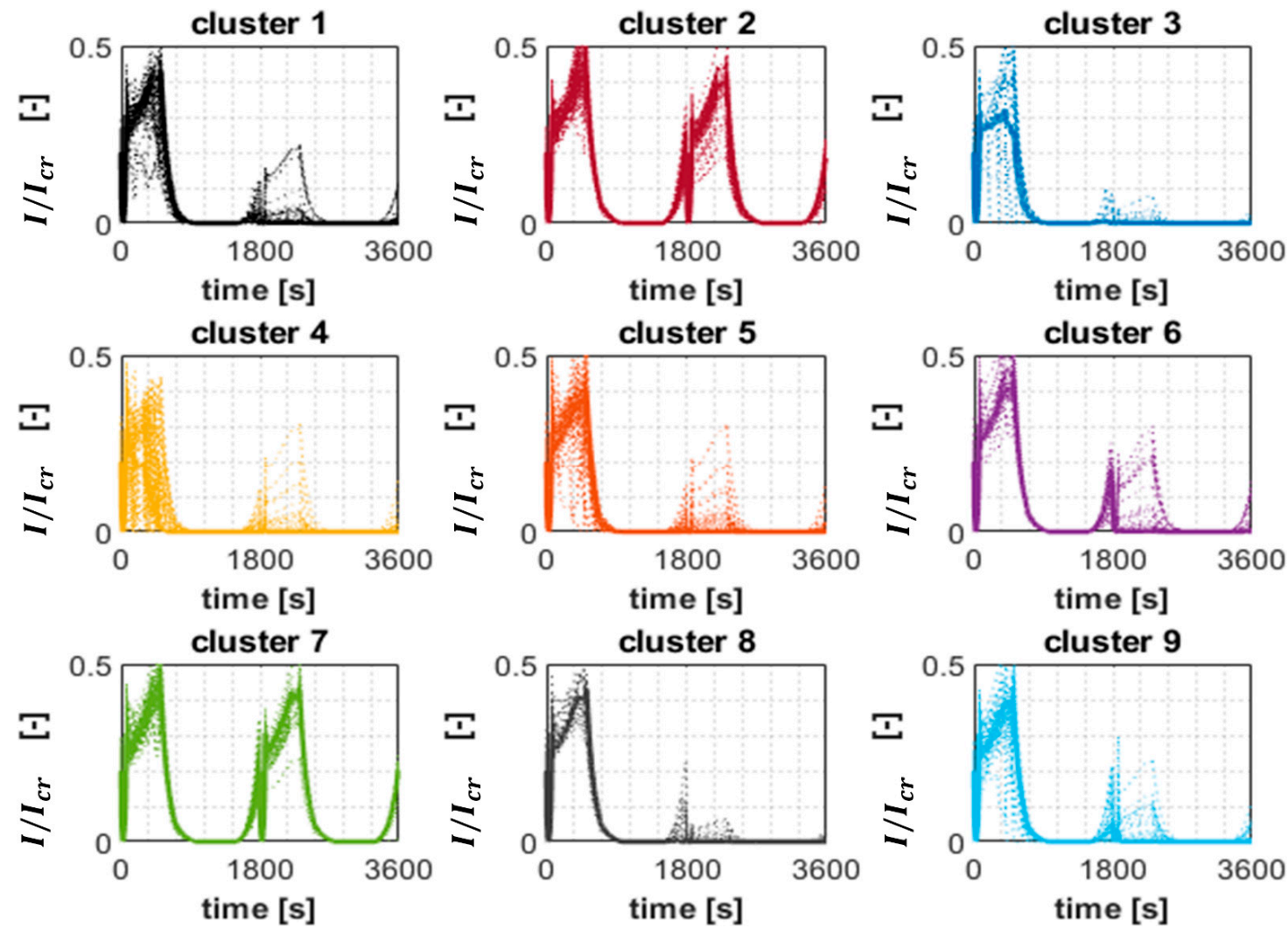

Figure A4. Nine clusters of $I / I_{c r}$ transient scenarios generated by POD-based Kriging metamodels. 


\section{References}

1. ITER-The Way to New Energy. Available online: https://www.iter.org/ (accessed on 5 October 2020).

2. Bigot, B. ITER construction and manufacturing progress toward first plasma. Fusion Eng. Des. 2018, 146, 124-129. [CrossRef]

3. Takahashi, Y.; Yoshida, K.; Nabara, Y.; Edaya, M.; Mitchell, N. Simulation of Quench Tests of the Central Solenoid Insert Coil in the ITER Central Solenoid Model Coil. IEEE Trans. Appl. Supercond. 2006, 16, 783-786. [CrossRef]

4. Mitchell, N.; Bessette, D.; Gallix, R.; Jong, C.; Knaster, J.; Libeyre, P.; Sborchia, C.; Simon, F. The ITER magnet system. IEEE Trans. Appl. Supercond. 2008, 18, 435-440. [CrossRef]

5. Zanino, R.; Bessette, D.; Richard, L.S. Quench analysis of an ITER TF coil. Fusion Eng. Des. 2010, 85, 752-760. [CrossRef]

6. Perrault, D. Safety issues to be taken into account in designing future nuclear fusion facilities. Fusion Eng. Des. 2016, 109-111, 1733-1738. [CrossRef]

7. Lukacs, M.; Williams, L.G. Nuclear safety issues for fusion power plants. Fusion Eng. Des. 2019, 150, 111377. [CrossRef]

8. Lukacs, M.; Williams, L. A sensitivity analysis of the factors that influence the hazard potential of fusion power plants. Fusion Eng. Des. 2021, 164, 112183. [CrossRef]

9. Lomonaco, G.; Mainardi, E.; Marková, T.; Mazzini, G. Approaching Nuclear Safety Culture in Fission and Fusion Technology. Appl. Sci. 2021, 11, 4511. [CrossRef]

10. Perrault, D. Nuclear safety aspects on the road towards fusion energy. Fusion Eng. Des. 2018, 146, 130-134. [CrossRef]

11. Porfiri, M.T.; Taylor, N.; Ciattaglia, S.; Jin, X.Z.; Johnston, J.; Colling, B.; Eade, T.; Carloni, D.; Pinna, T.; Urbonavicius, E.; et al. Safety assessment for EU DEMO_Achievements and open issues in view of a generic site safety report. Fusion Eng. Des. 2020, 155, 111541. [CrossRef]

12. Taylor, N.; Ciattaglia, S.; Boyer, H.; Coombs, D.; Jin, X.Z.; Liger, K.; Mora, J.C.; Mazzini, G.; Pinna, T.; Urbonavičius, E. Resolving safety issues for a demonstration fusion power plant. Fusion Eng. Des. 2017, 124, 1177-1180. [CrossRef]

13. Taylor, N.; Cortes, P. Lessons learnt from ITER safety \& licensing for DEMO and future nuclear fusion facilities. Fusion Eng. Des. 2014, 89, 1995-2000. [CrossRef]

14. Taylor, N.P. Safety and licensing of nuclear facilities for fusion. In Proceedings of the 2015 IEEE 26th Symposium on Fusion Engineering (SOFE), Austin, TX, USA, 31 May-4 June 2015; pp. 1-8.

15. Wang, Z.; Chen, S.; Ge, D.; Chen, C.; Chen, Z. A quantitative risk assessment methodology based on CCDF risk curve for ac-cidental releases from fusion reactors. Nucl. Mater. Energy 2020, 25, 100819. [CrossRef]

16. Mitchell, N.; Devred, A.; Libeyre, P.; Lim, B.; Savary, F. The ITER Magnets: Design and Construction Status. IEEE Trans. Appl. Supercond. 2011, 22, 4200809. [CrossRef]

17. Froio, A.; Barucca, L.; Ciattaglia, S.; Cismondi, F.; Savoldi, L.; Zanino, R. Analysis of the effects of primary heat transfer system isolation valves in case of in-vessel loss-of-coolant accidents in the EU DEMO. Fusion Eng. Des. 2020, 159, 111926. [CrossRef]

18. Pesetti, A.; Marini, A.; Raucci, M.; Giambartolomei, G.; Olcese, M.; Sarkar, B.; Aquaro, D. Large scale experimental facility for performance assessment of the vacuum vessel pressure suppression system of ITER. Fusion Eng. Des. 2021, 171, 112523. [CrossRef]

19. Lioce, D.; Orlandi, S.; Moteleb, M.; Ciampichetti, A.; Afzali, L.; Ghirelli, N.; Guo, B.; Tomasello, M.; Whitted, D.; Giammei, M.; et al. ITER Tokamak Cooling Water System Design Status. Fusion Sci. Technol. 2019, 75, 841-848. [CrossRef]

20. Galleni, F.; Moscardini, M.; Eboli, M.; Del Nevo, A.; Martelli, D.; Forgione, N. Preliminary analysis of an in-box LOCA in the breeding unit of the WCLL TBM for the ITER reactor with SIMMER-IV code. Fusion Eng. Des. 2021, 169, 112472. [CrossRef]

21. Moscardini, M.; Galleni, F.; Pucciarelli, A.; Eboli, M.; Del Nevo, A.; Paci, S.; Forgione, N. Thermo-hydraulic analysis of PbLi ancillary system of WCLL TBM undergoing in-box LOCA. Fusion Eng. Des. 2021, 168, 112614. [CrossRef]

22. Rivas, J.; Dies, J.; Fajarnés, X. Revisiting the analysis of passive plasma shutdown during an ex-vessel loss of coolant accident in ITER blanket. Fusion Eng. Des. 2015, 98-99, 2206-2209. [CrossRef]

23. Vicini, L.; Schiliuk, N.; Coscarelli, E.; Dell'Orco, G.; Narcisi, V.; Caruso, G. Thermal hydraulic transient analysis of ITER safe-ty-relevant secondary cooling water system. Fusion Eng. Des. 2021, 165, 112244. [CrossRef]

24. Du, Q.; Liu, C.; Ding, K.; Lu, K.; Long, Y.; Han, Q.; Wang, J.; Song, Y.; Niu, E.; Bauer, P. Test Facility and Results of ITER PF4 Current Leads. IEEE Trans. Plasma Sci. 2020, 48, 1428-1431. [CrossRef]

25. Du, Q.; Liu, C.; Ding, K.; Lu, K.; Song, Y.; Wang, J.; Han, Q.; Dong, Y.; Niu, E.; Bauer, P. Cold Performance Tests of the ITER 68 kA HTS Current Lead Prototypes. Fusion Eng. Des. 2020, 163, 112114. [CrossRef]

26. Nicolás-Pérez, F.; Velasco, F.J.S.; García-Cascales, J.R.; Otón-Martínez, R.A.; Bentaib, A.; Chaumeix, N. Evaluation of different models for turbulent combustion of hydrogen-air mixtures. Large Eddy Simulation of a LOVA sequence with hydrogen def-lagration in ITER Vacuum Vessel. Fusion Eng. Des. 2020, 161, 111901. [CrossRef]

27. Zanino, R.; Bonifetto, R.; Brighenti, A.; Isono, T.; Ozeki, H.; Savoldi, L. Prediction, experimental results and analysis of the ITER TF insert coil quench propagation tests, using the 4C code. Supercond. Sci. Technol. 2017, 31, 035004. [CrossRef]

28. Bellaera, R.; Bonifetto, R.; Di Maio, F.; Pedroni, N.; Savoldi, L.; Zanino, R.; Zio, E. Integrated deterministic and probabilistic safety assessment of a superconducting magnet cryogenic cooling circuit for nuclear fusion applications. Reliab. Eng. Syst. Saf. 2020, 201, 106945. [CrossRef]

29. IAEA. ITER Technical Basis, ITER EDA Documentation Series No. 24. IAEA: Vienna, Austria, 2002. Available online: https: //www-pub.iaea.org/MTCD/Publications /PDF/ITER-EDA-DS-24.pdf (accessed on 5 October 2020). 
30. ITER. ITER_D_22HV5L, ITER: Design Description Document: DDD 11 Magnet. 2006. Available online: https://industryportal. f4e.europa.eu/IP_WS_DOCUMENTS_TMP/16/_F4E-OPE-086_[6]\%20Design\%20Description\%20Document\%20(DDD)\%2 0DDD11-1\%20Engineering\%20Description.pdf (accessed on 5 October 2020).

31. Savoldi, L.; Bonifetto, R.; Pedroni, N.; Zanino, R. Analysis of a Protected Loss of Flow Accident (LOFA) in the ITER TF Coil Cooling Circuit. IEEE Trans. Appl. Supercond. 2017, 28, 1-9. [CrossRef]

32. Wu, Y.; Chen, Z.; Hu, L.; Jin, M.; Li, Y.; Jiang, J.; Yu, J.; Alejaldre, C.; Stevens, E.; Kim, K.; et al. Identification of safety gaps for fusion demonstration reactors. Nat. Energy 2016, 1, 16154. [CrossRef]

33. Aldemir, T. A survey of dynamic methodologies for probabilistic safety assessment of nuclear power plants. Ann. Nucl. Energy 2013, 52, 113-124. [CrossRef]

34. Karanki, D.; Rahman, S.; Dang, V.; Zerkak, O. Epistemic and aleatory uncertainties in integrated deterministic and probabilistic safety assessment: Tradeoff between accuracy and accident simulations. Reliab. Eng. Syst. Saf. 2017, 162, 91-102. [CrossRef]

35. Richard, L.S.; Casella, F.; Fiori, B.; Zanino, R. The 4C code for the cryogenic circuit conductor and coil modeling in ITER. Cryogenics 2010, 50, 167-176. [CrossRef]

36. Simpson, T.W.; Peplinski, J.D.; Koch, P.N.; Allen, J.K. Metamodels for computer-based engineering design: Survey and recommendations. Eng. Comput. 2001, 17, 129-150. [CrossRef]

37. Echard, B.; Gayton, N.; Lemaire, M. AK-MCS: An active learning reliability method combining Kriging and Monte Carlo Simulation. Struct. Saf. 2011, 33, 145-154. [CrossRef]

38. Schöbi, R.; Sudret, B.; Marelli, S. Rare Event Estimation Using Polynomial-Chaos Kriging. ASCE-ASME J. Risk Uncertain. Eng. Syst. Part A Civ. Eng. 2017, 3, D4016002. [CrossRef]

39. Turati, P.; Pedroni, N.; Zio, E. Simulation-based exploration of high-dimensional system models for identifying unexpected events. Reliab. Eng. Syst. Saf. 2017, 165, 317-330. [CrossRef]

40. Turati, P.; Cammi, A.; Lorenzi, S.; Pedroni, N.; Zio, E. Adaptive simulation for failure identification in the Advanced Lead Fast Reactor European Demonstrator. Prog. Nucl. Energy 2018, 103, 176-190. [CrossRef]

41. Wang, G.G.; Shan, S. Review of Metamodeling Techniques in Support of Engineering Design Optimization. J. Mech. Des. 2006, 129, 370-380. [CrossRef]

42. Marrel, A.; Perot, N.; Mottet, C. Development of a surrogate model and sensitivity analysis for spatio-temporal numerical simulators. Stoch. Environ. Res. Risk Assess. 2014, 29, 959-974. [CrossRef]

43. Nanty, S.; Helbert, C.; Marrel, A.; Pérot, N.; Prieur, C. Uncertainty quantification for functional dependent random variables. Comput. Stat. 2016, 32, 559-583. [CrossRef]

44. Baraldi, P.; Di Maio, F.; Zio, E. Unsupervised Clustering for Fault Diagnosis in Nuclear Power Plant Components. Int. J. Comput. Intell. Syst. 2013, 6, 764-777. [CrossRef]

45. Galushin, S.; Kudinov, P. Scenario Grouping and Classification Methodology for Postprocessing of Data Generated by Integrated Deterministic-Probabilistic Safety Analysis. Sci. Technol. Nucl. Install. 2015, 2015, 1-13. [CrossRef]

46. Grishchenko, D.; Galushin, S.; Kudinov, P. Failure domain analysis and uncertainty quantification using surrogate models for steam explosion in a Nordic type BWR. Nucl. Eng. Des. 2019, 343, 63-75. [CrossRef]

47. Di Maio, F.; Vagnoli, M.; Zio, E. Transient identification by clustering based on Integrated Deterministic and Probabilistic Safety Analysis outcomes. Ann. Nucl. Energy 2016, 87, 217-227. [CrossRef]

48. Destino, V.; Bonifetto, R.; Di Maio, F.; Pedroni, N.; Zanino, R.; Zio, E. Identification of LOFA Precursors in ITER Supercon-ducting Magnet Cryogenic Cooling Circuit. Reliab. Eng. Syst. Saf. 2021, 209, 107426. [CrossRef]

49. Park, Y.-J.; Fan, S.-K.S.; Hsu, C.-Y. A Review on Fault Detection and Process Diagnostics in Industrial Processes. Processes 2020, 8, 1123. [CrossRef]

50. Abid, A.; Khan, M.T.; Iqbal, J. A review on fault detection and diagnosis techniques: Basics and beyond. Artif. Intell. Rev. 2020, 54, 3639-3664. [CrossRef]

51. Lei, Y.; Lin, J.; He, Z.; Zuo, M.J. A review on empirical mode decomposition in fault diagnosis of rotating machinery. Mech. Syst. Signal Pr. 2013, 35, 108-126. [CrossRef]

52. Li, W.; Li, H.; Gu, S.; Chen, T. Process fault diagnosis with model- and knowledge-based approaches: Advances and oppor-tunities. Control. Eng. Pract. 2020, 105, 104637. [CrossRef]

53. Hu, Y.; Baraldi, P.; Di Maio, F.; Zio, E. A Systematic Semi-Supervised Self-adaptable Fault Diagnostics approach in an evolving environment. Mech. Syst. Signal Process. 2017, 88, 413-427. [CrossRef]

54. Hu, Y.; Baraldi, P.; Di Maio, F.; Liu, J.; Zio, E. A method for fault diagnosis in evolving environment using unlabeled data. Proc. Inst. Mech. Eng. Part O J. Risk Reliab. 2020, 235, 33-49. [CrossRef]

55. Jalayer, M.; Orsenigo, C.; Vercellis, C. Fault detection and diagnosis for rotating machinery: A model based on convolutional LSTM, Fast Fourier and continuous wavelet transforms. Comput. Ind. 2020, 125, 103378. [CrossRef]

56. Chiosa, R.; Piscitelli, M.S.; Capozzoli, A. A Data Analytics-Based Energy Information System (EIS) Tool to Perform Meter-Level Anomaly Detection and Diagnosis in Buildings. Energies 2021, 14, 237. [CrossRef]

57. Termite, M.R.; Baraldi, P.; Al-Dahidi, S.; Bellani, L.; Compare, M.; Zio, E. A Never-Ending Learning Method for Fault Diagnostics in Energy Systems Operating in Evolving Environments. Energies 2019, 12, 4802. [CrossRef]

58. Tolo, S.; Tian, X.; Bausch, N.; Becerra, V.; Santhosh, T.V.; Vinod, G.; Patelli, E. Robust on-line diagnosis tool for the early ac-cident detection in nuclear power plants. Reliab. Eng. Syst. Saf. 2019, 186, 110-119. [CrossRef] 
59. Utah, M.; Jung, J. Fault state detection and remaining useful life prediction in AC powered solenoid operated valves based on traditional machine learning and deep neural networks. Nucl. Eng. Technol. 2020, 52, 1998-2008. [CrossRef]

60. Saeed, H.A.; Wang, H.; Peng, M.; Hussain, A.; Nawaz, A. Online fault monitoring based on deep neural network \& sliding window technique. Prog. Nucl. Energy 2020, 121, 103236. [CrossRef]

61. Yellapu, V.S.; Vajpayee, V.; Tiwari, A.P. Online Fault Detection and Isolation in Advanced Heavy Water Reactor Using Mul-tiscale Principal Component Analysis. IEEE Trans. Nucl. Sci. 2019, 66, 1790-1803. [CrossRef]

62. Wang, H.; Peng, M.-J.; Yu, Y.; Saeed, H.; Hao, C.-M.; Liu, Y.-K. Fault identification and diagnosis based on KPCA and similarity clustering for nuclear power plants. Ann. Nucl. Energy 2020, 150, 107786. [CrossRef]

63. Farber, J.A.; Cole, D.G. Detecting loss-of-coolant accidents without accident-specific data. Prog. Nucl. Energy 2020, 128, 103469. [CrossRef]

64. Min, J.H.; Kim, D.-W.; Park, C.-Y. Demonstration of the validity of the early warning in online monitoring system for nuclear power plants. Nucl. Eng. Des. 2019, 349, 56-62. [CrossRef]

65. Nguyen, T.N.; Downar, T.; Vilim, R. A probabilistic model-based diagnostic framework for nuclear engineering systems. Ann. Nucl. Energy 2020, 149, 107767. [CrossRef]

66. Wang, Z.; Xia, H.; Peng, B.; Yang, B.; Zhu, S.; Zhang, J.; Annor-Nyarko, M. A multi-stage hybrid fault diagnosis approach for operating conditions of nuclear power plant. Ann. Nucl. Energy 2020, 153, 108015. [CrossRef]

67. Al-Dahidi, S.; Di Maio, F.; Baraldi, P.; Zio, E.; Seraoui, R. A framework for reconciliating data clusters from a fleet of nuclear power plants turbines for fault diagnosis. Appl. Soft Comput. 2018, 69, 213-231. [CrossRef]

68. Richard, L.S.; Bonifetto, R.; Carli, S.; Froio, A.; Foussat, A.; Zanino, R. Artificial Neural Network (ANN) modeling of the pulsed heat load during ITER CS magnet operation. Cryogenics 2014, 63, 231-240. [CrossRef]

69. Froio, A.; Bonifetto, R.; Carli, S.; Quartararo, A.; Savoldi, L.; Zanino, R. Design and optimization of Artificial Neural Networks for the modelling of superconducting magnets operation in tokamak fusion reactors. J. Comput. Phys. 2016, 321, 476-491. [CrossRef]

70. ITER. ITER_D_K7G8GN v2.1, Central Interlock System Strategy for ITER Magnet Protection: Machine Protection Functions. 24 January 2014.

71. Feng, Z.; Liang, M.; Chu, F. Recent advances in time-frequency analysis methods for machinery fault diagnosis: A review with application examples. Mech. Syst. Signal Process. 2013, 38, 165-205. [CrossRef]

72. Li, B.; Zhang, P.-L.; Wang, Z.-J.; Mi, S.-S.; Liu, D.-S. A weighted multi-scale morphological gradient filter for rolling element bearing fault detection. ISA Trans. 2011, 50, 599-608. [CrossRef]

73. Lataniotis, C.; Wicaksono, D.; Marelli, S.; Sudret, B. UQLAB User Manual_Kriging (Gaussian Process Modelling); Report \# UQLab-V1.4-105; Risk, Safety and Uncertainty Quantification, ETH Zurich: Zürich, Switzerland, 2021. Available online: https:/ / uqftp.ethz.ch/uqlab_doc_pdf/1.4.0/UserManual_Kriging.pdf (accessed on 5 March 2021).

74. Wall, M.E.; Rechtsteiner, A.; Rocha, L.M. Singular Value Decomposition and Principal Component Analysis. In A Practical Approach to Microarray Data Analysis; Berrar, D.P., Dubitzky, W., Granzow, M., Eds.; Springer: Boston, MA, USA, 2003 ; pp. 91-109.

75. Von Luxburg, U. A tutorial on spectral clustering. Stat. Comput. 2007, 17, 395-416. [CrossRef]

76. Mandelli, D.; Smith, C.; Yilmaz, A.; Aldemir, T. Mining nuclear transient data through symbolic conversion. In Proceedings of the ANS PSA 2013 International Topical Meeting on Probabilistic Safety Assessment and Analysis, Columbia, SC, USA, 22-26 September 2013; American Nuclear Society: LaGrange Park, IL, USA, 2013.

77. Zio, E.; Di Maio, F.; Stasi, M. A data-driven approach for predicting failure scenarios in nuclear systems. Ann. Nucl. Energy 2010, 37, 482-491. [CrossRef]

78. Bezdec, J.C. Pattern Recognition with Fuzzy Objective Function Algorithms; Plenum Press: New York, NY, USA, 1981.

79. Baraldi, P.; Di Maio, F.; Rigamonti, M.; Zio, E.; Seraoui, R. Clustering for unsupervised fault diagnosis in nuclear turbine shut-down transients. Mech. Syst. Signal Process. 2015, 58-59, 160-178. [CrossRef] 